\title{
Palladium-Catalyzed Distannylation of ortho-Quinodimethanes
}

Hiroto Yoshida, * Saori Nakano, Yasuhito Yamaryo, Joji Ohshita, and Atsutaka Kunai*

Department of Applied Chemistry, Graduate School of Engineering, Hiroshima

University, Higashi-Hiroshima 739-8527, Japan.

\section{Supporting Information}

General Remarks. All manipulations of oxygen- and moisture-sensitive materials were conducted with a standard Schlenk technique under a purified argon atmosphere. Nuclear magnetic resonance spectra were taken on a JEOL EX-270 $\left({ }^{1} \mathrm{H}, 270 \mathrm{MHz} ;{ }^{13} \mathrm{C}\right.$, $67.8 \mathrm{MHz})$ spectrometer or a JEOL Lambda-400 $\left({ }^{1} \mathrm{H}, 400 \mathrm{MHz} ;{ }^{13} \mathrm{C}, 99.5 \mathrm{MHz} ;{ }^{119} \mathrm{Sn}\right.$, 147.5 MHz) spectrometer using residual chloroform $\left({ }^{1} \mathrm{H}\right)$ or $\mathrm{CDCl}_{3}\left({ }^{13} \mathrm{C}\right)$ as an internal standard and tetramethyltin $\left({ }^{119} \mathrm{Sn}\right)$ as an external standard. The preparative recycling gel permeation chromatography was performed with GL Science PU 614 equipped with Shodex GPC H-2001L and -2002L columns (chloroform as an eluent). Column chromatography was carried out using Merck Aluminium oxide 90, activated, neutral. Unless otherwise noted, commercially available reagents were used without purification. THF was distilled from sodium/benzophenone ketyl and purified by passing through activated aluminium oxide column $(5 \mathrm{~g} / 8 \mathrm{~mL}$ of THF) prior to use. MeCN was distilled from phosphorus pentoxide. 18-Crown-6 was recrystallized from distilled MeCN. KF (spray-dried) was vacuum dried at $100{ }^{\circ} \mathrm{C}$ for $12 \mathrm{~h}$.

\section{Diels-Alder Reaction of ortho-Quinodimethane Using 1a as a Precursor.}

Generation of ortho-quinodimethane from 1a has been confirmed by the Diels-Alder reaction with methyl acrylate.

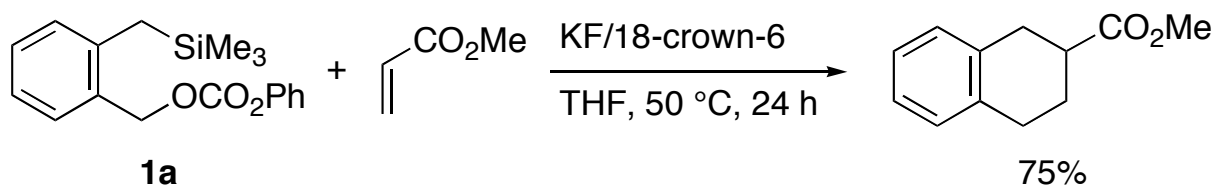

Distannylation Using Various Precursors or Ligands. Distannylation of $o$ quinodimethane with 2 using various precursors or ligands was examined. The results were summarized in Table A. 
Table A

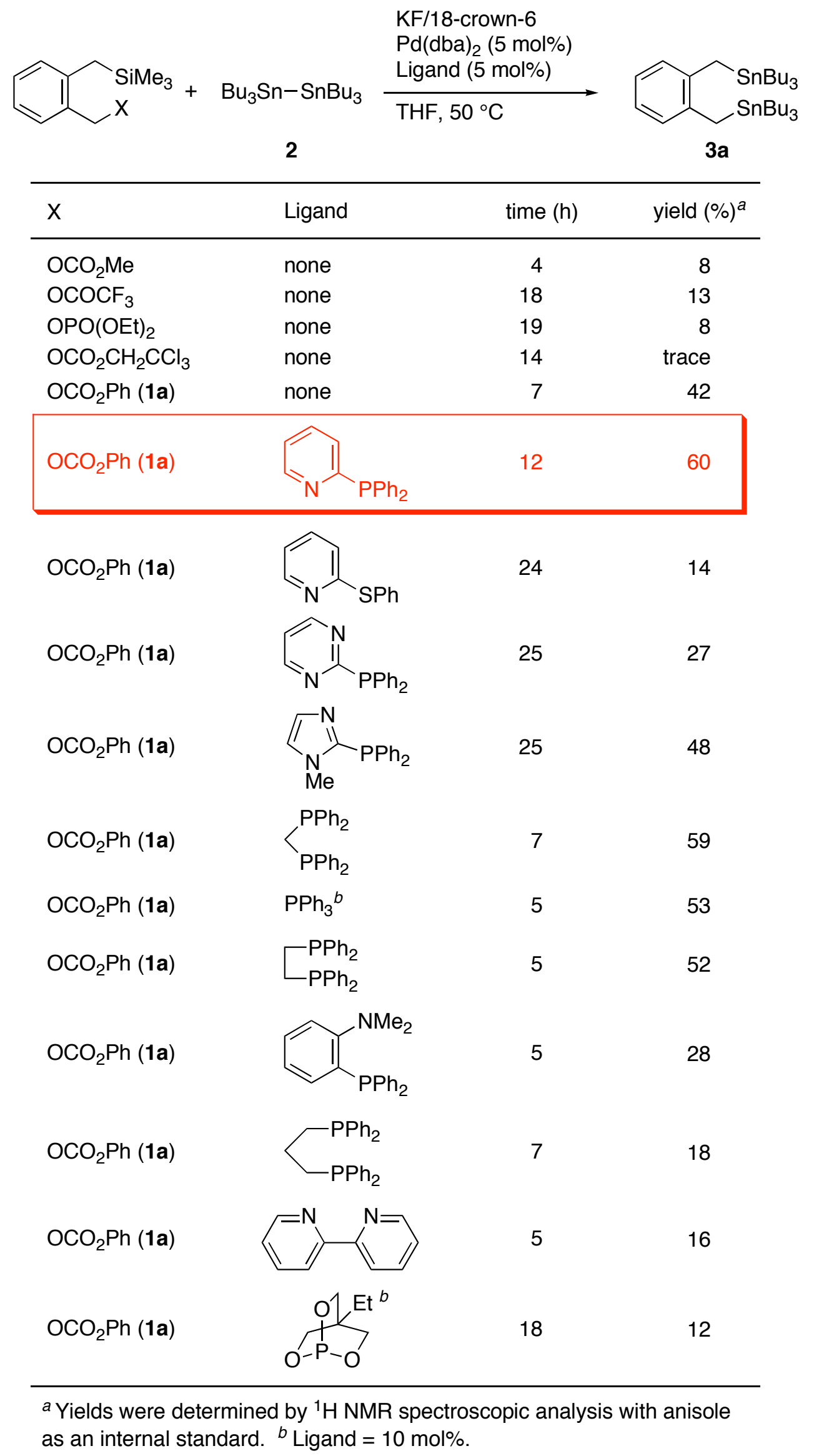


$o$-Quinodimethane Precursors. $o$-Quinodimethane precursors were synthesized from 2-(hydroxymethyl)-1-[(trimethylsilyl)methyl] $\operatorname{arenes}^{1,2}$ as follows.

Synthesis of Benzyl phenyl carbonate 1. To a $\mathrm{CH}_{2} \mathrm{Cl}_{2}$ solution $(20 \mathrm{~mL})$ of a 2(hydroxymethyl)-1-[(trimethylsilyl)methyl]arene (0.010 mol) were added pyridine $(1.6 \mathrm{~g}$, $0.020 \mathrm{~mol})$ and phenyl chloroformate $(2.3 \mathrm{~g}, 0.015 \mathrm{~mol})$ at $0{ }^{\circ} \mathrm{C}$, and the mixture was stirred at $0{ }^{\circ} \mathrm{C}$. After the completion of the reaction, the mixture was washed with water, and the resulting organic phase was dried over anhydrous $\mathrm{MgSO}_{4}$. Evaporation of the solvent followed by column chromatography gave $\mathbf{1}$.

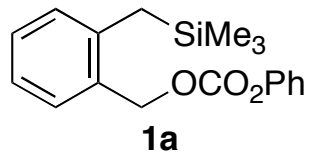

2-[(Trimethylsilyl)methyl]benzyl phenyl carbonate (1a). Isolated in $95 \%$ yield as a colorless oil: ${ }^{1} \mathrm{H} \mathrm{NMR}\left(\mathrm{CDCl}_{3}\right) \delta 0.03$ $(\mathrm{s}, 9 \mathrm{H}), 2.23(\mathrm{~s}, 2 \mathrm{H}), 5.24(\mathrm{~s}, 2 \mathrm{H}), 7.06(\mathrm{~d}, J=7.7 \mathrm{~Hz}, 1 \mathrm{H}), 7.12(\mathrm{t}$, $J=7.5 \mathrm{~Hz}, 1 \mathrm{H}), 7.18(\mathrm{~d}, J=8.5 \mathrm{~Hz}, 2 \mathrm{H}), 7.21-7.32(\mathrm{~m}, 2 \mathrm{H}), 7.38(\mathrm{t}, J=7.5 \mathrm{~Hz}, 3 \mathrm{H})$; ${ }^{13} \mathrm{C} \mathrm{NMR}\left(\mathrm{CDCl}_{3}\right) \delta-1.4,23.3,68.8,121.1,124.5,126.0,128.8,129.4,129.5,130.2$, 131.1, 140.0, 151.2, 153.8; Anal. Calcd for $\mathrm{C}_{18} \mathrm{H}_{22} \mathrm{O}_{3} \mathrm{Si}$ : C, 68.75; H, 7.05. Found: C, 68.58; H, 7.00.

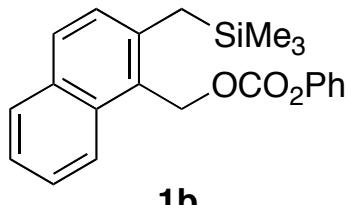

\section{\{2-[(Trimethylsilyl)methyl] naphth-1-yl\}methyl phenyl} carbonate (1b). Isolated in $81 \%$ yield as a pale yellow oil: ${ }^{1} \mathrm{H}$ NMR $\left(\mathrm{CDCl}_{3}\right) \delta 0.04(\mathrm{~s}, 9 \mathrm{H}), 2.53(\mathrm{~s}, 2 \mathrm{H}), 5.75$ (s, $\left.2 \mathrm{H}\right), 7.10-$ $7.32(\mathrm{~m}, 4 \mathrm{H}), 7.32-7.50(\mathrm{~m}, 3 \mathrm{H}), 7.56(\mathrm{td}, J=8.5,1.4 \mathrm{~Hz}, 1 \mathrm{H})$, $7.75(\mathrm{~d}, J=8.4 \mathrm{~Hz}, 1 \mathrm{H}), 7.82(\mathrm{~d}, J=8.0 \mathrm{~Hz}, 1 \mathrm{H}), 8.11(\mathrm{~d}, J=8.7 \mathrm{~Hz}, 1 \mathrm{H}) ; \quad{ }^{13} \mathrm{C} \mathrm{NMR}$ $\left(\mathrm{CDCl}_{3}\right) \delta$-1.5, 25.2, 64.4, 121.1, 123.4, 124.6, 126.0, 126.9, 128.5, 128.7, 129.4, 129.4, 129.6, 131.6, 133.2, 139.8, 151.2, 154.0; Anal. Calcd for $\mathrm{C}_{22} \mathrm{H}_{24} \mathrm{O}_{3} \mathrm{Si}$ : C, 72.49; $\mathrm{H}$, 6.64. Found: C, 72.20; H, 6.50.

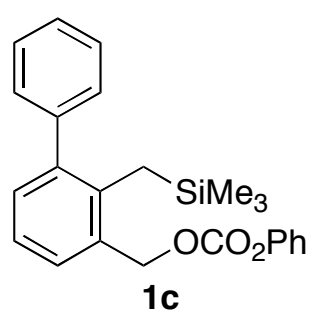

\section{3-Phenyl-2-[(trimethylsilyl) methyl]benzyl phenyl carbonate} (1c). Isolated in $88 \%$ yield as a pale yellow oil: ${ }^{1} \mathrm{H}$ NMR $\left(\mathrm{CDCl}_{3}\right) \delta-0.27(\mathrm{~s}, 9 \mathrm{H}), 2.42(\mathrm{~s}, 2 \mathrm{H}), 5.30$ (s, $\left.2 \mathrm{H}\right), 7.09-7.55$ (m, $13 \mathrm{H}) ;{ }^{13} \mathrm{C} \mathrm{NMR}\left(\mathrm{CDCl}_{3}\right) \delta-0.7,19.5,69.5,121.1,124.0,126.0$, 126.9, 128.2, 129.2, 129.5, 129.8, 131.1, 131.7, 138.5, 141.6, 142.5, 151.2, 153.8; Anal. Calcd for $\mathrm{C}_{24} \mathrm{H}_{26} \mathrm{O}_{3} \mathrm{Si}$ : C, 73.81; H, 6.71.

Found: $\quad$ C, 73.61; H, 6.45.<smiles>COCc1cccc(C)c1COc1ccccc1</smiles>

$1 d$
6-Methyl-2-[(trimethylsilyl)methyl]benzyl phenyl carbonate (1d). Isolated in $91 \%$ yield as a colorless oil: ${ }^{1} \mathrm{H}$ NMR $\left(\mathrm{CDCl}_{3}\right)$ $\delta 0.02(\mathrm{~s}, 9 \mathrm{H}), 2.30$ (s, $2 \mathrm{H}), 2.45$ (s, $3 \mathrm{H}), 5.34$ (s, $2 \mathrm{H}), 6.91$ (d, $J=$ 
$7.5 \mathrm{~Hz}, 1 \mathrm{H}), 6.98(\mathrm{~d}, J=7.5 \mathrm{~Hz}, 1 \mathrm{H}), 7.08-7.33(\mathrm{~m}, 4 \mathrm{H}), 7.34-7.46(\mathrm{~m}, 2 \mathrm{H}) ; \quad{ }^{13} \mathrm{C}$ NMR $\left(\mathrm{CDCl}_{3}\right) \delta-1.5,20.0,23.9,65.3,121.0,126.0,126.7,127.5,128.8,129.2,129.4$, 139.0, 141.2, 151.2, 153.9; Anal. Calcd for $\mathrm{C}_{19} \mathrm{H}_{24} \mathrm{O}_{3} \mathrm{Si}$ : C, 69.47; H, 7.36. Found: C, 69.38; H, 7.37.

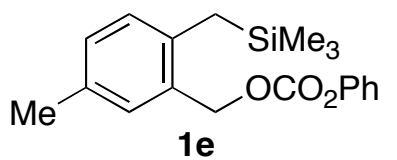

5-Methyl-2-[( trimethylsilyl) methyl]benzyl

phenyl

carbonate (1e). Isolated in $88 \%$ yield as a colorless oil: ${ }^{1} \mathrm{H}$

NMR $\left(\mathrm{CDCl}_{3}\right) \delta 0.02(\mathrm{~s}, 9 \mathrm{H}), 2.29(\mathrm{~s}, 2 \mathrm{H}), 2.44(\mathrm{~s}, 3 \mathrm{H}), 5.33$

(s, $2 \mathrm{H}), 6.90(\mathrm{~d}, J=7.7 \mathrm{~Hz}, 1 \mathrm{H}), 6.97(\mathrm{~d}, J=7.5 \mathrm{~Hz}, 1 \mathrm{H}), 7.07-7.33$ (m, $4 \mathrm{H}), 7.33-7.47$ $(\mathrm{m}, 2 \mathrm{H}) ;{ }^{13} \mathrm{C} \mathrm{NMR}\left(\mathrm{CDCl}_{3}\right) \delta-1.5,20.7,22.6,68.8,121.0,125.9,129.3,129.4,129.5$, 130.8, 133.7, 136.6, 151.2, 153.7; Anal. Calcd for $\mathrm{C}_{19} \mathrm{H}_{24} \mathrm{O}_{3} \mathrm{Si}$ : C, 69.47; H, 7.36. Found: $\quad$ C, 69.31; H, 7.54.<smiles>COCc1cc(C)cc(C)c1COc1ccccc1</smiles>

4,6-Dimethyl-2-[(trimethylsilyl)methyl]benzyl phenyl carbonate (1f). Isolated in $91 \%$ yield as a pale yellow oil: ${ }^{1} \mathrm{H} \mathrm{NMR}\left(\mathrm{CDCl}_{3}\right) \delta 0.04$ (s, $\left.9 \mathrm{H}\right), 2.28$ (s, $\left.2 \mathrm{H}\right), 2.29$ (s, $\left.3 \mathrm{H}\right)$, 2.42 (s, $3 \mathrm{H}), 5.32$ (s, $2 \mathrm{H}), 6.74$ (s, $1 \mathrm{H}), 6.82$ (s, $1 \mathrm{H}), 7.10-$ $7.34(\mathrm{~m}, 3 \mathrm{H}), 7.34-7.49(\mathrm{~m}, 2 \mathrm{H}) ;{ }^{13} \mathrm{C} \mathrm{NMR}\left(\mathrm{CDCl}_{3}\right) \delta$-1.5, 19.9, 21.1, 23.8, 65.2, 121.1, 125.9, 126.4, 127.7, 128.1, 129.4, 138.5, 138.8, 141.0, 151.2, 154.0; Anal. Calcd for $\mathrm{C}_{20} \mathrm{H}_{26} \mathrm{O}_{3} \mathrm{Si}$ : C, 70.13; H, 7.65. Found: C, 70.40; H, 7.69.<smiles>CC(C)Cc1cc(F)ccc1COOc1ccccc1</smiles>

$1 \mathrm{~g}$

\section{4-Fluoro-2-[( trimethylsilyl) methyl]benzyl}

carbonate (1g). Isolated in $96 \%$ yield as a pale yellow crystal: ${ }^{1} \mathrm{H} \mathrm{NMR}\left(\mathrm{CDCl}_{3}\right) \delta 0.55$ (s, $\left.9 \mathrm{H}\right), 2.24(\mathrm{~s}, 2 \mathrm{H}), 5.20(\mathrm{~s}, 2 \mathrm{H})$, 6.67-6.87 (m, $2 \mathrm{H}), 7.10-7.22(\mathrm{~m}, 2 \mathrm{H}), 7.22-7.32(\mathrm{~m}, 1 \mathrm{H}), 7.32-7.47(\mathrm{~m}, 3 \mathrm{H}) ;{ }^{13} \mathrm{C}$ $\operatorname{NMR}\left(\mathrm{CDCl}_{3}\right) \delta-1.5,23.7,68.0,111.3\left(\mathrm{~d}, J_{\mathrm{C}-\mathrm{F}}=22.0 \mathrm{~Hz}\right), 115.7\left(\mathrm{~d}, J_{\mathrm{C}-\mathrm{F}}=20.7 \mathrm{~Hz}\right)$, $121.0,126.0,129.5,132.3\left(\mathrm{~d}, J_{\mathrm{C}-\mathrm{F}}=0.34 \mathrm{~Hz}\right), 143.1,143.2,153.7,151.1,162.9\left(\mathrm{~d}, J_{\mathrm{C}-\mathrm{F}}=\right.$ 246.6 Hz); Anal. Calcd for $\mathrm{C}_{18} \mathrm{H}_{21} \mathrm{FO}_{3} \mathrm{Si}$ : C, 65.03; H, 6.37. Found: C, 65.22; H, 6.32 .

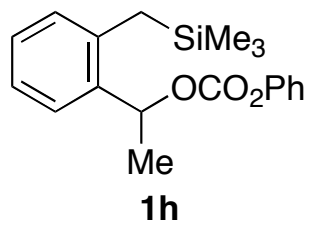

\section{Q-Methyl-2-[(trimethylsilyl) methyl]benzyl phenyl carbonate}

(1h). Isolated in $98 \%$ yield as a pale yellow oil: ${ }^{1} \mathrm{H}$ NMR $\left(\mathrm{CDCl}_{3}\right) \delta 0.03(\mathrm{~s}, 9 \mathrm{H}), 1.66(\mathrm{~d}, J=6.5 \mathrm{~Hz}, 3 \mathrm{H}), 2.13(\mathrm{~d}, J=14.0$ $\mathrm{Hz}, 1 \mathrm{H}), 2.31(\mathrm{~d}, J=14.0 \mathrm{~Hz}, 1 \mathrm{H}), 6.02(\mathrm{q}, J=6.5 \mathrm{~Hz}, 1 \mathrm{H}), 7.01$ $(\mathrm{dd}, J=7.2,1.9 \mathrm{~Hz}, 1 \mathrm{H}), 7.12-7.32(\mathrm{~m}, 5 \mathrm{H}), 7.32-7.46(\mathrm{~m}, 2 \mathrm{H}), 7.49$ (dd, $J=7.2,1.9$ $\mathrm{Hz}, 1 \mathrm{H}) ; \quad{ }^{13} \mathrm{C} \mathrm{NMR}\left(\mathrm{CDCl}_{3}\right) \delta-1.4,21.7,23.1,74.3,121.1,124.8,125.9,126.1,127.9$, 129.4, 129.6, 136.9, 137.7, 151.2, 153.2; Anal. Calcd for $\mathrm{C}_{19} \mathrm{H}_{24} \mathrm{O}_{3} \mathrm{Si}$ : C, 69.47; H, 7.36. Found: C, 69.30; H, 7.40. 


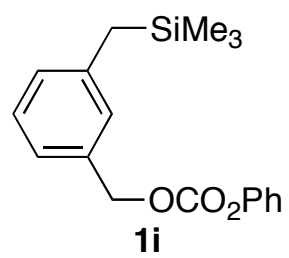

3-[(Trimethylsilyl)methyl]benzyl phenyl carbonate (1i). Isolated in 94\% yield as a yellow oil: ${ }^{1} \mathrm{H} \mathrm{NMR}\left(\mathrm{CDCl}_{3}\right) \delta 0.02(\mathrm{~s}, 9$ H), 2.13 (s, $2 \mathrm{H}), 5.25$ (s, $2 \mathrm{H}), 7.02$ (d, J= $7.7 \mathrm{~Hz}, 1 \mathrm{H}), 7.09$ (s, $1 \mathrm{H})$, 7.16-7.34 (m, $5 \mathrm{H}), 7.34-7.48(\mathrm{~m}, 2 \mathrm{H}) ;{ }^{13} \mathrm{C} \mathrm{NMR}\left(\mathrm{CDCl}_{3}\right) \delta-1.9$, 27.0, 70.6, 121.1, 124.2, 126.0, 128.1, 128.4, 128.5, 129.5, 134.6, 141.1, 151.2, 153.7; Anal. Calcd for $\mathrm{C}_{18} \mathrm{H}_{22} \mathrm{O}_{3} \mathrm{Si}$ : C, 68.75; H, 7.05. Found: C, $68.60 ; \mathrm{H}, 7.09$.

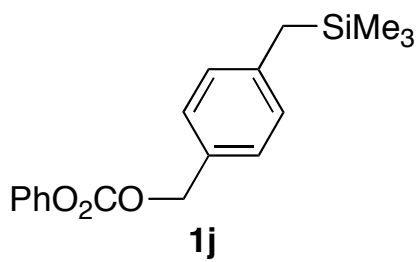

4-[(Trimethylsilyl)methyl]benzyl phenyl carbonate (1j). Isolated in $97 \%$ yield as a orange crystal: ${ }^{1} \mathrm{H} \mathrm{NMR}\left(\mathrm{CDCl}_{3}\right)$ $\delta-0.01(\mathrm{~s}, 9 \mathrm{H}), 2.10(\mathrm{~s}, 2 \mathrm{H}), 5.21(\mathrm{~s}, 2 \mathrm{H}), 7.02$ (d, $J=7.9$ $\mathrm{Hz}, 2 \mathrm{H}), 7.09-7.45(\mathrm{~m}, 7 \mathrm{H}) ;{ }^{13} \mathrm{C} \mathrm{NMR}\left(\mathrm{CDCl}_{3}\right) \delta-2.0$, 27.0, 70.5, 121.0, 126.0, 128.2, 128.8, 129.4, 130.1, 141.5,

151.2, 153.7; Anal. Calcd for $\mathrm{C}_{18} \mathrm{H}_{22} \mathrm{O}_{3} \mathrm{Si}$ : C, 68.75; H, 7.05. Found: C, 69.00; $\mathrm{H}, 7.12$.

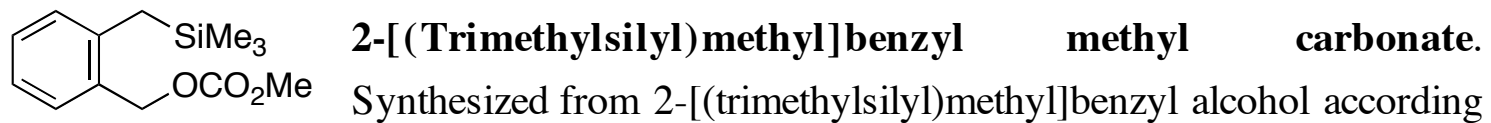
to a literature method. ${ }^{3} \quad$ Isolated in $73 \%$ yield as a colorless oil: b.p. $120{ }^{\circ} \mathrm{C}$ (bath temp., $1 \mathrm{mmHg}) ; \quad{ }^{1} \mathrm{H}$ NMR $\left(\mathrm{CDCl}_{3}\right) \delta 0.02(\mathrm{~s}, 9 \mathrm{H}), 2.10$ (s, $\left.3 \mathrm{H}\right), 2.17$ (s, $\left.2 \mathrm{H}\right), 5.07$ (s, $\left.2 \mathrm{H}\right)$, $7.03(\mathrm{~d}, J=7.4 \mathrm{~Hz}, 1 \mathrm{H}), 7.09(\mathrm{td}, J=7.4,1.3 \mathrm{~Hz}, 1 \mathrm{H}), 7.22(\mathrm{td}, J=7.4,1.7 \mathrm{~Hz}, 1 \mathrm{H})$, $7.33(\mathrm{~d}, J=7.4 \mathrm{~Hz}, 1 \mathrm{H}) ; \quad{ }^{13} \mathrm{C} \mathrm{NMR}\left(\mathrm{CDCl}_{3}\right) \delta-1.5,23.1,54.8,68.0,124.3,128.5$, 129.3, 129.8, 131.4, 139.7, 155.8; Anal. Calcd for $\mathrm{C}_{13} \mathrm{H}_{20} \mathrm{O}_{3} \mathrm{Si}$ : C, 61.87; H, 7.99. Found: $\quad$ C, 61.64; H, 8.05.

$\begin{array}{ll}\mathrm{SiMe}_{3} & \begin{array}{l}\text { 2-[(Trimethylsilyl) methyl]benzyl trifluoroacetate. To a } \\ \mathrm{OCOCF}_{3}\end{array} \\ \mathrm{CH}_{2} \mathrm{Cl}_{2} \text { solution }(20 \mathrm{~mL}) \text { of 2-[(trimethylsilyl)methyl]benzyl alcohol }\end{array}$ $(0.010 \mathrm{~mol})$ were added triethylamine $(2.0 \mathrm{~g}, 0.020 \mathrm{~mol})$ and trifluoroacetic anhydride $(3.2$ $\mathrm{g}, 0.015 \mathrm{~mol}$ ) at $0{ }^{\circ} \mathrm{C}$, and the mixture was stirred at room temperature for $3 \mathrm{~h}$ before evaporation of the solvent. The residue was treated with hexane and the insoluble material was removed by filtration. Evaporation of the solvent gave 2[(trimethylsilyl)methyl]benzyl trifluoroacetate (3), which was used for the reaction without further purification. Isolated in $95 \%$ yield as a pale yellow oil: ${ }^{1} \mathrm{H}$ NMR $\left(\mathrm{CDCl}_{3}\right)$

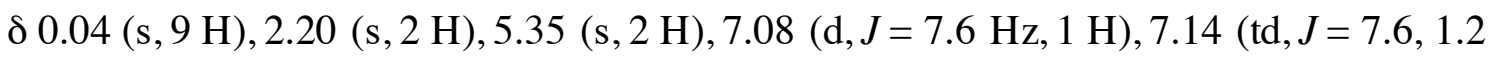
$\mathrm{Hz}, 1 \mathrm{H}), 7.28(\mathrm{td}, J=7.6,1.5 \mathrm{~Hz}, 1 \mathrm{H}), 7.34(\mathrm{dd}, J=7.6,1.2 \mathrm{~Hz}, 1 \mathrm{H}) ;{ }^{13} \mathrm{C} \mathrm{NMR}$ $\left(\mathrm{CDCl}_{3}\right) \delta$-1.6, 23.2, 68.0, $114.6\left(\mathrm{q}, J_{\mathrm{C}-\mathrm{F}}=285.5 \mathrm{~Hz}\right), 124.6,129.3,129.6,130.3,140.2$, 
$157.5\left(\mathrm{q}, J_{\mathrm{C}-\mathrm{F}}=41.8 \mathrm{~Hz}\right) ;$ Anal. Calcd for $\mathrm{C}_{13} \mathrm{H}_{17} \mathrm{~F}_{3} \mathrm{O}_{2} \mathrm{Si}: \quad$ C, 53.78; H, 5.90 . Found: C, 53.78; H, 5.94.

$\mathrm{SiMe}_{3}$ Diethyl 2-[(trimethylsilyl)methyl]benzyl phosphate. To a alcohol (0.39 g, $2.0 \mathrm{mmol})$ were added pyridine $(0.24 \mathrm{~g}, 3.0 \mathrm{mmol})$ and diethyl chlorophosphate $(0.52 \mathrm{mg}, 3.0 \mathrm{mmol})$ at $0{ }^{\circ} \mathrm{C}$, and the mixture was stirred at $0{ }^{\circ} \mathrm{C}$ for $3 \mathrm{~h}$. After the reaction mixture was washed with water, the resulting organic phase was dried over anhydrous $\mathrm{MgSO}_{4}$, filtered, and concentrated in vacuo. Silica gel column chromatography gave diethyl 2-[(trimethylsilyl)methyl]benzyl phosphate (4) in 54\% yield as a colorless oil: ${ }^{1} \mathrm{H}$ NMR $\left(\mathrm{CDCl}_{3}\right) \delta-0.01(\mathrm{~s}, 9 \mathrm{H}), 1.26(\mathrm{td}, J=7.5,1.0 \mathrm{~Hz}, 6 \mathrm{H})$, 2.04 (s, 2 H), 4.00-4.17 (m, 4 H), 5.09 (d, J= 7.5 Hz, 2 H), 7.01 (d, J=7.7 Hz, $1 \mathrm{H}), 7.09$ $(\mathrm{td}, J=7.5,1.0 \mathrm{~Hz}, 1 \mathrm{H}), 7.21(\mathrm{td}, J=7.5,1.2 \mathrm{~Hz}, 1 \mathrm{H}), 7.36(\mathrm{~d}, J=7.5 \mathrm{~Hz}, 1 \mathrm{H}) ;{ }^{13} \mathrm{C}$ $\operatorname{NMR}\left(\mathrm{CDCl}_{3}\right) \delta-1.6,16.0\left(\mathrm{~d}, J_{\mathrm{C}-\mathrm{P}}=6.6 \mathrm{~Hz}\right), 22.9,63.7\left(\mathrm{~d}, J_{\mathrm{C}-\mathrm{P}}=5.7 \mathrm{~Hz}\right), 67.3\left(\mathrm{~d}, J_{\mathrm{C}-\mathrm{P}}=\right.$ $5.7 \mathrm{~Hz}), 124.3,128.4,129.2,129.2,132.3\left(\mathrm{~d}, J_{\mathrm{C}-\mathrm{P}}=7.4 \mathrm{~Hz}\right), 139.3$; Anal. Calcd for $\mathrm{C}_{15} \mathrm{H}_{27} \mathrm{O}_{4} \mathrm{PSi}: \quad \mathrm{C}, 54.52 ; \mathrm{H}, 8.24$. Found: $\mathrm{C}, 54.40 ; \mathrm{H}, 8.22$

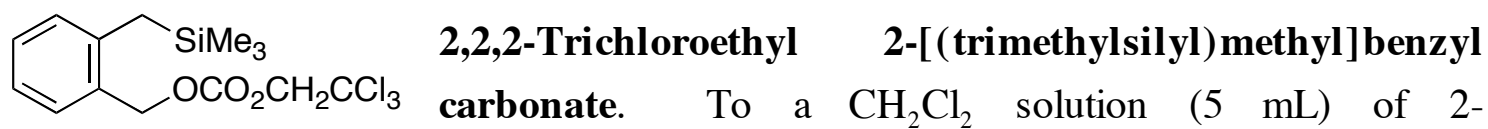
[(trimethylsilyl)methyl]benzyl alcohol $(0.39 \mathrm{~g}, 2.0 \mathrm{mmol})$ were added pyridine $(0.24 \mathrm{~g}, 3.0$ mmol) and 2,2,2-trichloroethyl chloroformate $(0.51 \mathrm{~g}, 2.4 \mathrm{mmol})$ at $0{ }^{\circ} \mathrm{C}$, and the mixture was stirred at $0{ }^{\circ} \mathrm{C}$ for $3 \mathrm{~h}$. After the reaction mixture was washed with sat. $\mathrm{NaHCO}_{3}$ aq, the resulting organic phase was dried over anhydrous $\mathrm{MgSO}_{4}$, filtered, and concentrated in vacuo. Silica gel column chromatography gave 2,2,2-trichloroethyl 2[(trimethylsilyl)methyl]benzyl carbonate (5) in $80 \%$ yield as a colorless oil: ${ }^{1} \mathrm{H}$ NMR $\left(\mathrm{CDCl}_{3}\right) \delta 0.02(\mathrm{~s}, 9 \mathrm{H}), 2.20(\mathrm{~s}, 2 \mathrm{H}), 4.78(\mathrm{~s}, 2 \mathrm{H}), 5.22(\mathrm{~s}, 2 \mathrm{H}), 7.04(\mathrm{~d}, J=7.5 \mathrm{~Hz}, 1 \mathrm{H})$, $7.10(\operatorname{td}, J=7.5,1.0 \mathrm{~Hz}, 1 \mathrm{H}), 7.18-7.32(\mathrm{~m}, 1 \mathrm{H}), 7.35(\mathrm{~d}, J=7.7 \mathrm{~Hz}, 1 \mathrm{H}) ; \quad{ }^{13} \mathrm{C} \mathrm{NMR}$ $\left(\mathrm{CDCl}_{3}\right) \delta$-1.5, 23.2, 69.2, 76.8, 94,4, 124.4, 128.9, 129.4, 130.2, 130.8, 140.1, 154.0; Anal. Calcd for $\mathrm{C}_{14} \mathrm{H}_{19} \mathrm{Cl}_{3} \mathrm{O}_{3} \mathrm{Si}$ : C, 45.48; H, 5.18. Found: C, 45.51; H, 5.16.

Distannylation of o-Quinodimethanes. A General Procedure. A THF solution (1.0 mL) of 18-crown-6 (0.16 g, $0.60 \mathrm{mmol})$, diphenyl-2-pyridylphosphine (2.6 mg, 0.010 $\mathrm{mmol})$ and $\mathrm{Pd}(\mathrm{dba})_{2}(5.8 \mathrm{mg}, 0.010 \mathrm{mmol})$ was degassed by two freeze-thaw cycles. To this solution were added 1 (0.30 mmol), 2 (0.12 g, $0.20 \mathrm{mmol})$ and KF (0.035 g, 0.60 $\mathrm{mmol}$ ), and the resulting mixture was stirred at $50^{\circ} \mathrm{C}$. After the time specified in Scheme 2, the mixture was diluted with ethyl acetate, filtered through a Celite plug, and concentrated. Alumina column chromatography (hexane as an eluent, Activity IV) 
followed by gel permeation chromatography (chloroform as an eluent) gave the corresponding product.

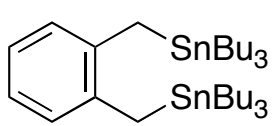

$3 \mathbf{a}$

$\alpha, \alpha$ '-Bis(tributylstannyl)-o-xylene (3a). Isolated in 55\% yield as a pale yellow oil: ${ }^{1} \mathrm{H}$ NMR $\left(\mathrm{CDCl}_{3}\right) \delta 0.65-1.04(\mathrm{~m}, 30 \mathrm{H}), 1.16-1.59$ $(\mathrm{m}, 24 \mathrm{H}), 2.14(\mathrm{~s}, 4 \mathrm{H}), 6.73-6.97(\mathrm{~m}, 4 \mathrm{H}) ;{ }^{13} \mathrm{C}$ NMR $\left(\mathrm{CDCl}_{3}\right)$

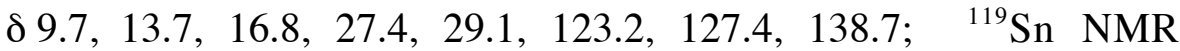

$\left(\mathrm{CDCl}_{3}\right) \delta$-12.4. Anal. Calcd for $\mathrm{C}_{32} \mathrm{H}_{62} \mathrm{Sn}_{2}$ : C, 56.17; $\mathrm{H}, 9.13$. Found: $\mathrm{C}$, $56.21 ; \mathrm{H}, 9.13$.<smiles>CCCCCCCCCCCc1ccc2ccccc2c1C[SbH2]C</smiles>

3b

1,2-Bis[( tributylstannyl)methyl] naphthalene $(\mathbf{3 b})$. Isolated in $63 \%$ yield as a pale yellow oil: ${ }^{1} \mathrm{H}$ NMR $\left(\mathrm{CDCl}_{3}\right) \delta 0.50-0.99$ $(\mathrm{m}, 30 \mathrm{H}), 1.02-1.61(\mathrm{~m}, 24 \mathrm{H}), 2.37$ (s, $2 \mathrm{H}), 2.60$ (s, $2 \mathrm{H}), 7.11$ (d, $J=8.3 \mathrm{~Hz}, 1 \mathrm{H}), 7.23-7.47(\mathrm{~m}, 3 \mathrm{H}), 7.72(\mathrm{~d}, J=7.6 \mathrm{~Hz}, 1 \mathrm{H}), 7.85$ $(\mathrm{d}, J=8.6 \mathrm{~Hz}, 1 \mathrm{H}) ;{ }^{13} \mathrm{C} \mathrm{NMR}\left(\mathrm{CDCl}_{3}\right) \delta 9.9,10.3,13.0,13.6$, $18.4,27.4,29.1,29.1,123.1,123.3,124.9,127.8,128.5,131.4,132.5,135.4 ;{ }^{119} \mathrm{Sn}$ NMR $\left(\mathrm{CDCl}_{3}\right) \delta-10.4,-7.1$; Anal. Calcd for $\mathrm{C}_{36} \mathrm{H}_{64} \mathrm{Sn}_{2}$ : C, 58.88; H, 8.78. Found: $\quad$ C, 58.70; H, 8.80.

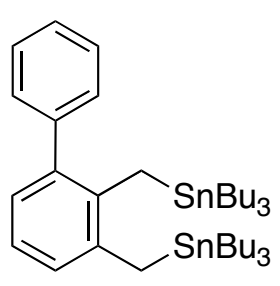

$3 c$

$\alpha, \alpha$ '-Bis(tributylstannyl)-3-phenyl-o-xylene (3c). Isolated in $59 \%$ yield as a pale yellow oil: ${ }^{1} \mathrm{H}$ NMR $\left(\mathrm{CDCl}_{3}\right) \delta 0.48-0.68(\mathrm{~m}, 6$ H), 0.72-0.98 (m, 24 H), 1.08-1.56 (m, 24 H), 2.18 (s, 2 H), 2.21 (s, 2 H), $6.76(\mathrm{dd}, J=7.1,1.6 \mathrm{~Hz}, 1 \mathrm{H}), 6.81-6.78(\mathrm{~m}, 2 \mathrm{H}), 7.16-7.33(\mathrm{~m}, 2$ $\mathrm{H}), 7.38(\mathrm{t}, J=7.4 \mathrm{~Hz}, 3 \mathrm{H}) ;{ }^{13} \mathrm{C} \mathrm{NMR}\left(\mathrm{CDCl}_{3}\right) \delta 9.9,10.2,13.6$, 13.7, 14.6, 18.0, 27.3, 27.5, 28.9, 29.1, 122.6, 125.1, 126.3, 126.5, 128.0, 129.4, 136.5, 139.4, 139.7, 143.8; ${ }^{119} \mathrm{Sn}$ NMR $\left(\mathrm{CDCl}_{3}\right) \delta-13.3,-13.1 ; \quad$ Anal. Calcd for $\mathrm{C}_{38} \mathrm{H}_{66} \mathrm{Sn}_{2}$ : C, 60.03; H, 8.75. Found: C, 60.07; H, 8.53.<smiles>CCCCCCCCCCCCCCCCCCC</smiles>
9.15 . $\delta$-13.5,-13.0; Anal. Calcd for $\mathrm{C}_{33} \mathrm{H}_{64} \mathrm{Sn}_{2}$ : C, 56.76; H, 9.24. Found: C, 56.70; $\mathrm{H}$,

$\alpha, \alpha^{\prime}$-Bis(tributylstannyl)-3-methyl-o-xylene (3d). Isolated in $62 \%$ yield as a pale yellow oil: ${ }^{1} \mathrm{H}$ NMR $\left(\mathrm{CDCl}_{3}\right) \delta 0.66-1.06(\mathrm{~m}, 30$ $\mathrm{H}), 1.14-1.60$ (m, $24 \mathrm{H}), 2.12$ (s, $2 \mathrm{H}), 2.14-2.27$ (m, $5 \mathrm{H}), 6.69-6.85$ $(\mathrm{m}, 3 \mathrm{H}) ; \quad{ }^{13} \mathrm{C} \mathrm{NMR}\left(\mathrm{CDCl}_{3}\right) \delta 9.7,10.3,13.7,13.9,17.5,21.2,27.4$, 29.1, 122.7, 125.0, 125.3, 133.1, 137.2, 138.6; ${ }^{119} \mathrm{Sn}$ NMR $\left(\mathrm{CDCl}_{3}\right)$

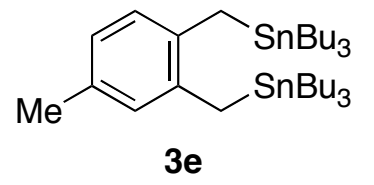

$\alpha, \alpha$ '-Bis(tributylstannyl)-4-methyl-o-xylene (3e). Isolated in 59\% yield as a pale yellow oil: ${ }^{1} \mathrm{H} \mathrm{NMR}\left(\mathrm{CDCl}_{3}\right) \delta \quad 0.57-1.02$ 
(m, $30 \mathrm{H}), 1.14-1.59(\mathrm{~m}, 24 \mathrm{H}), 2.11(\mathrm{~s}, 4 \mathrm{H}), 2.20(\mathrm{~s}, 3 \mathrm{H}), 6.64(\mathrm{~d}, J=7.6 \mathrm{~Hz}, 1 \mathrm{H}), 6.70$ $(\mathrm{s}, 1 \mathrm{H}), 6.77(\mathrm{~d}, J=7.6 \mathrm{~Hz}, 1 \mathrm{H}) ; \quad{ }^{13} \mathrm{C} \mathrm{NMR}\left(\mathrm{CDCl}_{3}\right) \delta 9.7,9.7,13.7,16.1,16.7,20.9$, 27.4, 29.1, 124.0, 127.4, 128.2, 132.3, 135.4, 138.5; ${ }^{119} \mathrm{Sn} \mathrm{NMR}\left(\mathrm{CDCl}_{3}\right) \delta$-13.6, -12.9; Anal. Calcd for $\mathrm{C}_{33} \mathrm{H}_{64} \mathrm{Sn}_{2}$ : C, 56.76; H, 9.24. Found: C, 56.70; H, 9.28.<smiles>CCCCCCCCCCCCCCCCCCC</smiles>

$3 f$ $\alpha, \alpha$-Bis(tributylstannyl) -3,5-dimethyl-o-xylene

(3f). 8 0.66-1.04 (m, $30 \mathrm{H}), 1.16-1.54(\mathrm{~m}, 24 \mathrm{H}), 2.07(\mathrm{~s}, 2 \mathrm{H}), 2.10$ $2.23(\mathrm{~m}, 8 \mathrm{H}), 6.57(\mathrm{~s}, 1 \mathrm{H}), 6.59(\mathrm{~s}, 1 \mathrm{H}) ;{ }^{13} \mathrm{C} \mathrm{NMR}\left(\mathrm{CDCl}_{3}\right)$ ठ 9.7, 10.3, 13.4, 13.6, 13.7, 17.4, 20.8, 21.1, 27.4, 29.1, 125.9, 125.0, 126.1, 131.6, 132.9, 138.4; ${ }^{119} \mathrm{Sn}$ NMR $\left(\mathrm{CDCl}_{3}\right) \delta-14.1 ;$ Anal. Calcd for $\mathrm{C}_{34} \mathrm{H}_{66} \mathrm{Sn}_{2}$ : C, 57.33; H, 9.34. Found: C, 57.54; H, 9.50 .

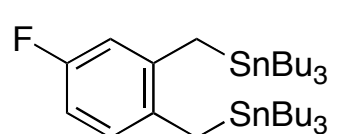

$3 \mathrm{~g}$

$\alpha, \alpha^{\prime}$-Bis(tributylstannyl)-4-fluoro-o-xylene (3g). Isolated in $50 \%$ yield as a pale yellow oil: ${ }^{1} \mathrm{H}$ NMR $\left(\mathrm{CDCl}_{3}\right) \delta 0.67-0.96(\mathrm{~m}$, $30 \mathrm{H}), 1.07-1.56(\mathrm{~m}, 24 \mathrm{H}), 2.08(\mathrm{~s}, 2 \mathrm{H}), 2.12(\mathrm{~s}, 2 \mathrm{H}), 6.53(\mathrm{td}, J=$ 8.4, 2.7 Hz, $1 \mathrm{H}), 6.60(\mathrm{dd}, J=10.4,2.7 \mathrm{~Hz}, 1 \mathrm{H}), 6.80(\mathrm{dd}, J=8.4$, $6.0 \mathrm{~Hz}, 1 \mathrm{H}) ; \quad{ }^{13} \mathrm{C} \mathrm{NMR}\left(\mathrm{CDCl}_{3}\right) \delta$ 9.7, 9.8, 13.6, 15.8, 17.4, 27.4, 29.0, 29.2, $109.8(\mathrm{~d}$, $\left.J_{\mathrm{C}-\mathrm{F}}=20.8 \mathrm{~Hz}\right), 113.3\left(\mathrm{~d}, J_{\mathrm{C}-\mathrm{F}}=20.7 \mathrm{~Hz}\right), 128.2\left(\mathrm{~d}, J_{\mathrm{C}-\mathrm{F}}=8.6 \mathrm{~Hz}\right), 134.2,141.0\left(\mathrm{~d}, J_{\mathrm{C}-\mathrm{F}}=\right.$ $7.3 \mathrm{~Hz}), 159.8\left(\mathrm{~d}, J_{\mathrm{C}-\mathrm{F}}=239.2 \mathrm{~Hz}\right) ;{ }^{119} \mathrm{Sn} \mathrm{NMR}\left(\mathrm{CDCl}_{3}\right) \delta-12.8\left(\mathrm{~d}, J_{\mathrm{Sn}-\mathrm{F}}=21.6 \mathrm{~Hz}\right),-$ $10.6\left(\mathrm{~d}, J_{\mathrm{Sn}-\mathrm{F}}=10.8 \mathrm{~Hz}\right)$; Anal. Calcd for $\mathrm{C}_{32} \mathrm{H}_{61} \mathrm{FSn}_{2}$ : C, 54.73; H, 8.76. Found: C, 54.71; H, 8.72.

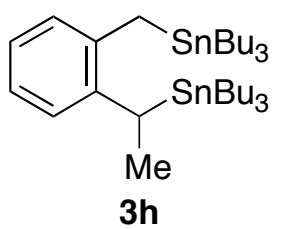

$3 \mathbf{h}$

$\alpha, \alpha$-Bis(tributylstannyl)- $\alpha$-methyl-o-xylene (3h). Isolated in $63 \%$ yield as a pale yellow oil: ${ }^{1} \mathrm{H}$ NMR $\left(\mathrm{CDCl}_{3}\right) \delta 0.50-1.05(\mathrm{~m}, 30$ H), 1.05-1.80 (m, $24 \mathrm{H}), 2.11(\mathrm{~s}, 1 \mathrm{H}), 2.15(\mathrm{~s}, 2 \mathrm{H}), 2.16(\mathrm{~s}, 3 \mathrm{H})$, 6.73-6.76 (m, $4 \mathrm{H}) ;{ }^{13} \mathrm{C} \mathrm{NMR}\left(\mathrm{CDCl}_{3}\right) \delta$ 9.1, 9.5, 13.7, 13.7, 16.8, 17.5, 23.5, 27.4, 27.5, 29.1, 29.1, 123.0, 123.5, 123.9, 127.4, 138.1, 143.9; ${ }^{119} \mathrm{Sn}$ NMR $\left(\mathrm{CDCl}_{3}\right) \delta-14.4,-6.6$; Anal. Calcd for $\mathrm{C}_{33} \mathrm{H}_{64} \mathrm{Sn}_{2}$ : C, 56.76; H, 9.24. Found: C, $56.64 ; \mathrm{H}, 9.08$.<smiles>IC1Cc2ccccc2C[IH]1</smiles>

7

Iododestannylation of 3a. To a $\mathrm{CH}_{2} \mathrm{Cl}_{2}$ solution $(1 \mathrm{~mL})$ of $\mathbf{3 a}(0.023 \mathrm{~g}$, $0.034 \mathrm{mmol})$ was added iodine $(0.018 \mathrm{~g}, 0.071 \mathrm{mmol})$, and the mixture was stirred at r.t. for $1.5 \mathrm{~h}$ before evaporation of the solvent. Silica gel column chromatography (hexane as an eluent) gave $7(5.2 \mathrm{mg}, 43 \% \text { yield })^{4}$ as a pale yellow solid: ${ }^{1} \mathrm{H} \mathrm{NMR}\left(\mathrm{CDCl}_{3}\right) \delta 4.58(\mathrm{~s}, 4 \mathrm{H}), 7.16-7.35(\mathrm{~m}, 4 \mathrm{H}) ;{ }^{13} \mathrm{C} \mathrm{NMR}\left(\mathrm{CDCl}_{3}\right) \delta$ 1.7, 129.0, $130.8,137.4$. 


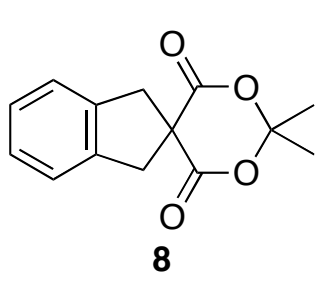

Synthesis of 8. Meldrum's acid $(0.014 \mathrm{~g}, 0.095 \mathrm{mmol})$ was added to a suspension of $\mathrm{NaH}(4.2 \mathrm{mg}, 0.095 \mathrm{mmol}, 55 \%$ dispersion in mineral oil) in DMSO $(0.2 \mathrm{~mL})$, and the mixture was stirred at r.t. for $0.5 \mathrm{~h}$. To this mixture was added a DMSO solution $(1 \mathrm{~mL})$ of crude 7, prepared as above [from 3a $(0.030 \mathrm{~g}, 0.043 \mathrm{mmol})$ and $\mathrm{I}_{2}$ $(0.023 \mathrm{~g}, 0.091 \mathrm{mmol})]$, and the resulting mixture was stirred at $50{ }^{\circ} \mathrm{C}$ for $13 \mathrm{~h}$. The mixture was poured into $0.04 \mathrm{M}$ aqueous $\mathrm{HCl}(25 \mathrm{~mL})$ and extracted with chloroform (3 times). The combined extracts were washed with $\mathrm{H}_{2} \mathrm{O}$, dried over $\mathrm{MgSO}_{4}$ and concentrated. Gel permeation chromatography gave $\mathbf{8}(2.9 \mathrm{mg}, 27 \% \text { yield based on } \mathbf{3 a})^{5}$ as a white solid: ${ }^{1} \mathrm{H} \mathrm{NMR}\left(\mathrm{CDCl}_{3}\right) \delta 1.83(\mathrm{~s}, 6 \mathrm{H}), 3.74(\mathrm{~s}, 4 \mathrm{H}), 7.23(\mathrm{brs}, 4 \mathrm{H}) ;{ }^{13} \mathrm{C}$ NMR $\left(\mathrm{CDCl}_{3}\right) \delta 29.0,45.6,52.4,105.2,124.0,127.6,138.8,170.5$.<smiles>CCOC(=O)C(Cc1ccccc1CC(C(=O)OCC)C(=O)OCC)C(=O)OCC</smiles>

Synthesis of 9. Diethyl malonate $(0.021 \mathrm{~g}, 0.13 \mathrm{mmol})$ was added to a suspension of $\mathrm{NaH}(5.7 \mathrm{mg}, 0.13 \mathrm{mmol}, 55 \%$ dispersion in mineral oil) in DMSO $(0.4 \mathrm{~mL})$, and the mixture was stirred at r.t. for $0.5 \mathrm{~h}$. To this mixture was added a DMSO solution $(1 \mathrm{~mL})$ of crude 7, prepared as above [from 3a $(0.036 \mathrm{~g}, 0.052 \mathrm{mmol})$ and $\mathrm{I}_{2}(0.028 \mathrm{~g}$, $0.11 \mathrm{mmol})]$, and the resulting mixture was stirred at r.t. for $19 \mathrm{~h}$. The mixture was poured into $0.04 \mathrm{M}$ aqueous $\mathrm{HCl}(25 \mathrm{~mL})$ and extracted with ether (3 times). The combined extracts were washed with $\mathrm{H}_{2} \mathrm{O}$, dried over $\mathrm{MgSO}_{4}$ and concentrated. Gel permeation chromatography gave 9 (3.2 mg, $15 \%$ yield based on 3a) ${ }^{6}$ as a pale yellow oil: ${ }^{1} \mathrm{H}$ NMR $\left(\mathrm{CDCl}_{3}\right) \delta 1.20(\mathrm{t}, J=$ $7.3 \mathrm{~Hz}, 12 \mathrm{H}), 3.28$ (d, $J=7.6 \mathrm{~Hz}, 4 \mathrm{H}), 3.69$ (t, $J=7.6 \mathrm{~Hz}, 2 \mathrm{H}), 4.15(\mathrm{q}, J=7.3 \mathrm{~Hz}, 8 \mathrm{H})$, $7.14(\mathrm{~s}, 4 \mathrm{H}) ;{ }^{13} \mathrm{C} \mathrm{NMR}\left(\mathrm{CDCl}_{3}\right) \delta 13.9,31.1,52.9,61.5,127.0,129.6,136.1,168.9$.

(1) Dai-Ho, G.; Mariano, P. S. J. Org. Chem. 1988, 53, 5113.

(2) (a) Lan, A. J. Y.; Heuckeroth, R. O.; Mariano, P. S. J. Am. Chem. Soc. 1987, 109, 2738. (b) Lenihan, B. D.; Shechter, H. J. Org. Chem. 1998, 63, 2072.

(3) (a) Minami, I.; Tsuji, J. Tetrahedron 1987, 43, 3903. (b) Oppolzer, W.; Robyr, C. Tetrahedron 1994, 50, 415.

(4) Rezende, D. de B.; Alcantara, M. R.; de Arruda Campos, I. P.; Toscano, V. G.; Ebeling, G.; Lopes, J. C. D. Tetrahedron 1997, 53, 10113.

(5) Ridvan, L.; Závada, J. Tetrahedron 1997, 53, 14793.

(6) Fakhri, S. A.; Yousefi, B. H. Tetrahedron 2000, 56, 8301. 


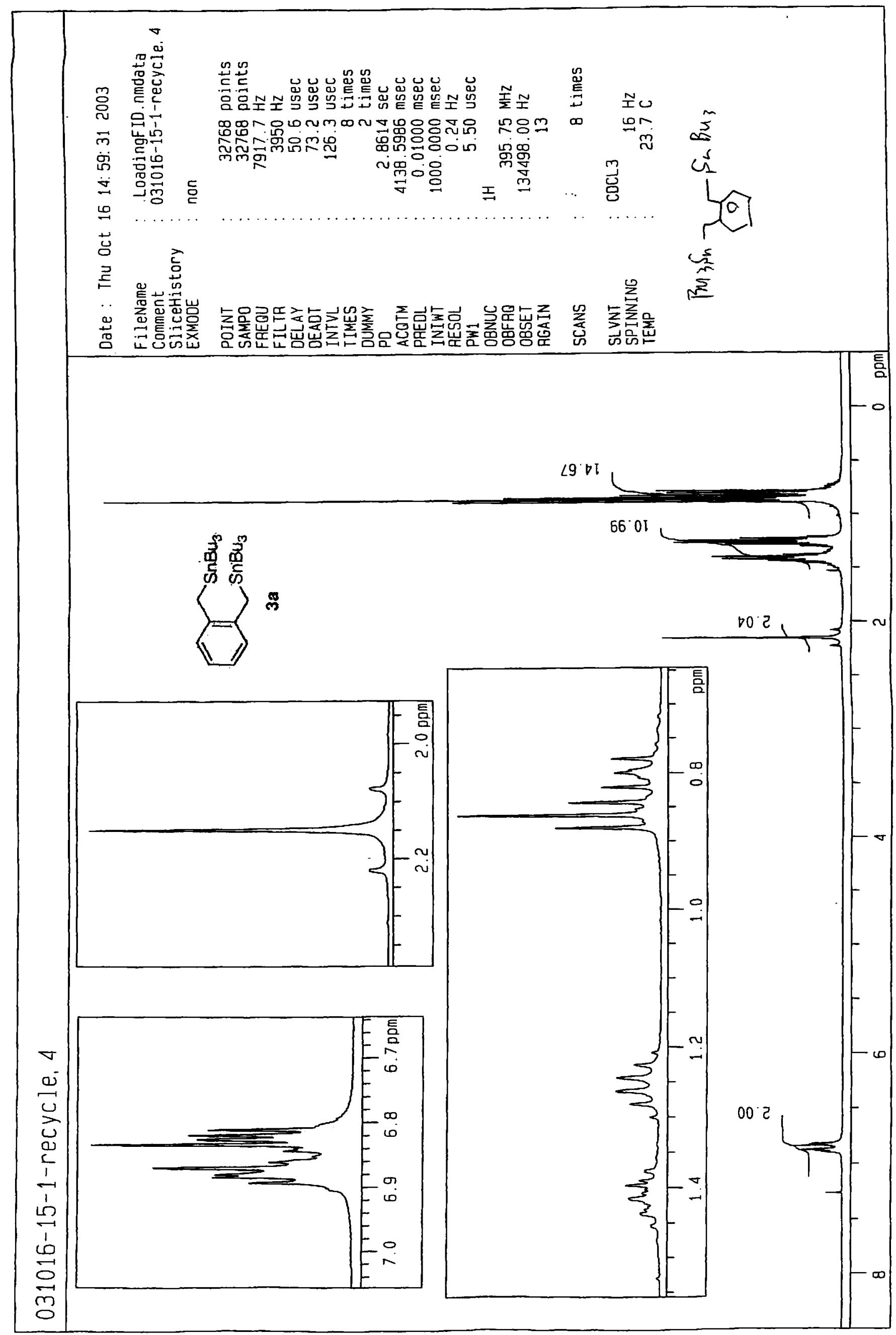




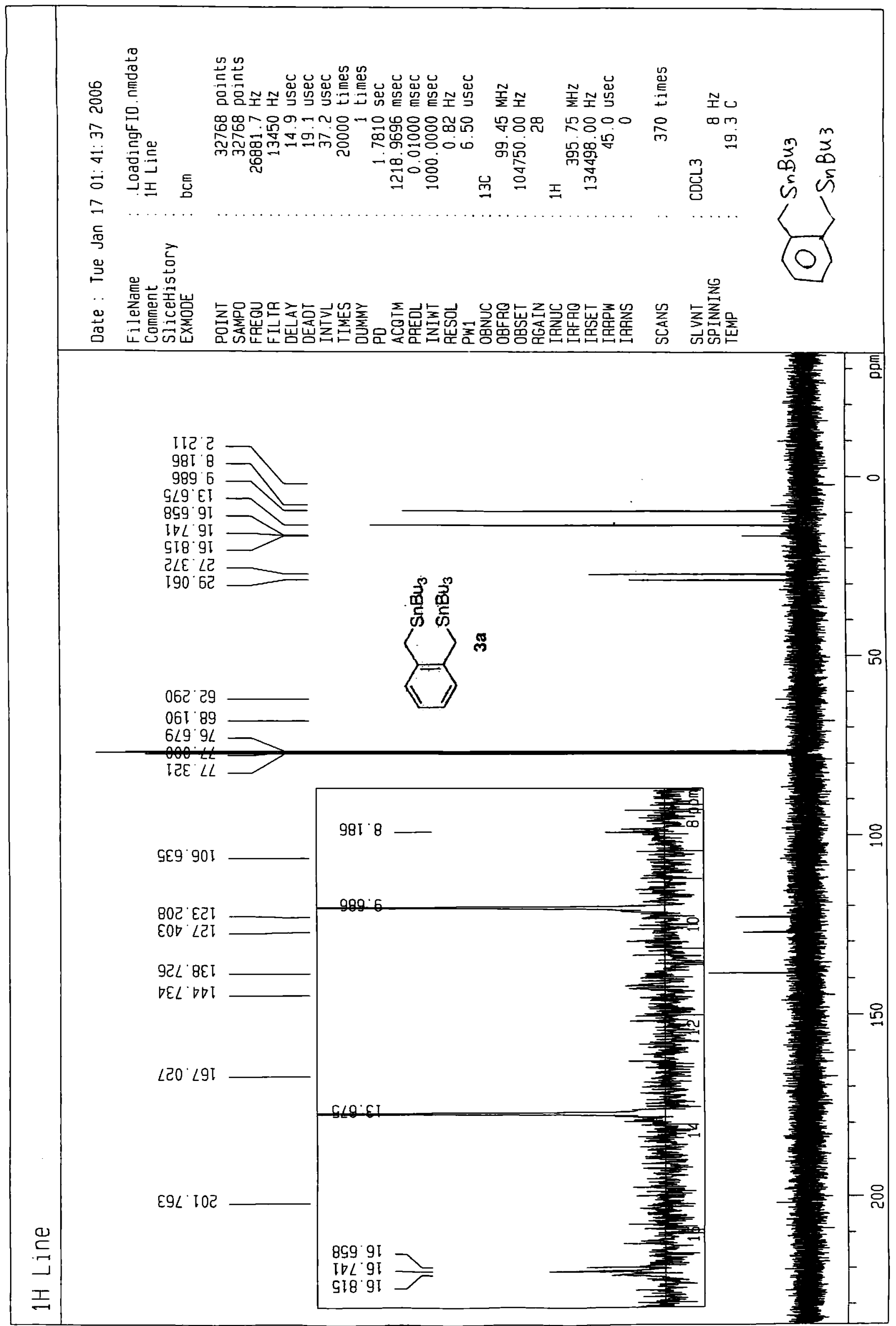


$m$

$\ddot{\circ}$

$\ddot{-j}$

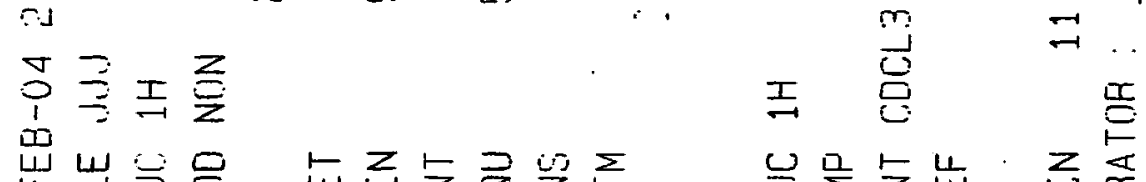

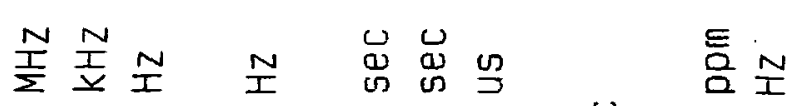

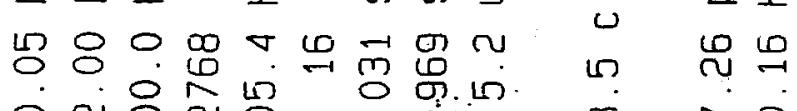

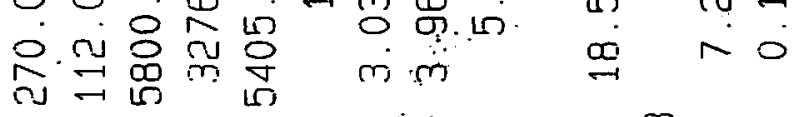

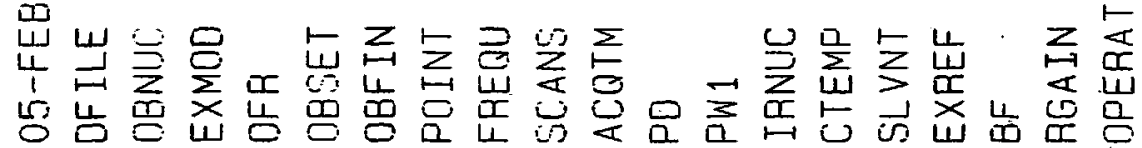

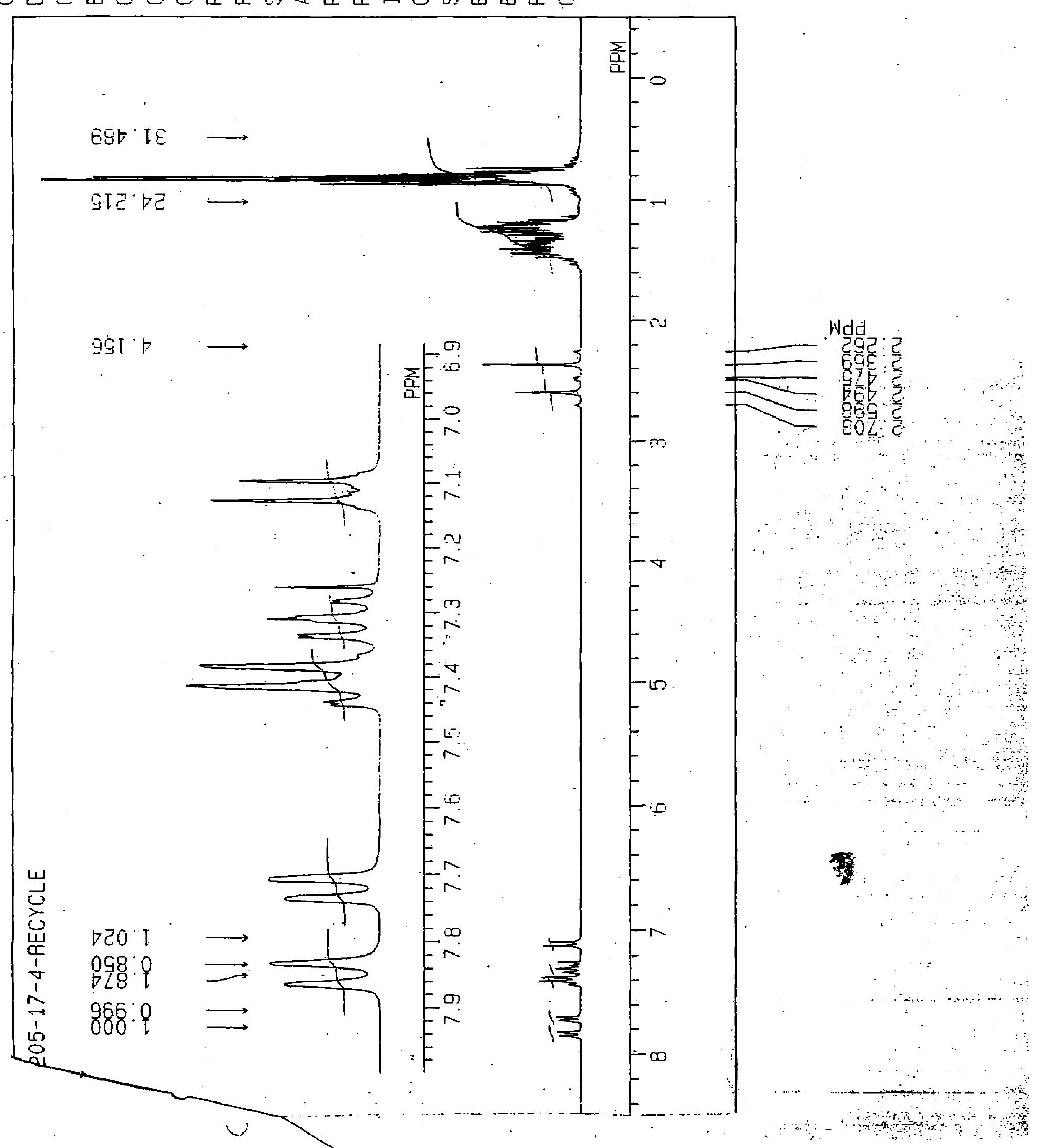

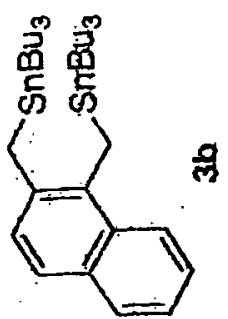




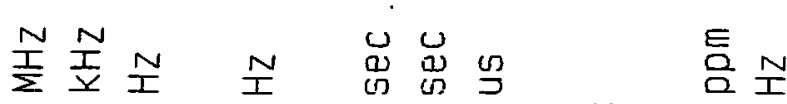

ه

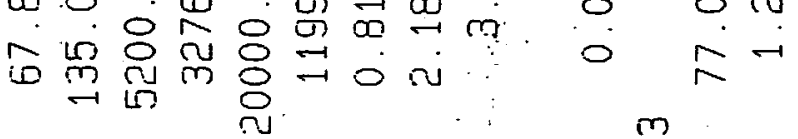

政

工 $\stackrel{m}{\stackrel{\mathrm{C}}{\mathrm{C}}}$

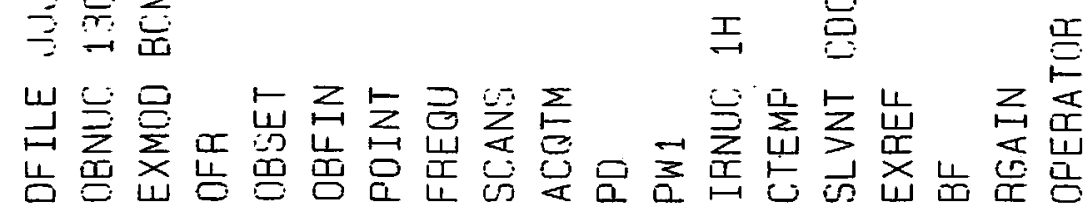
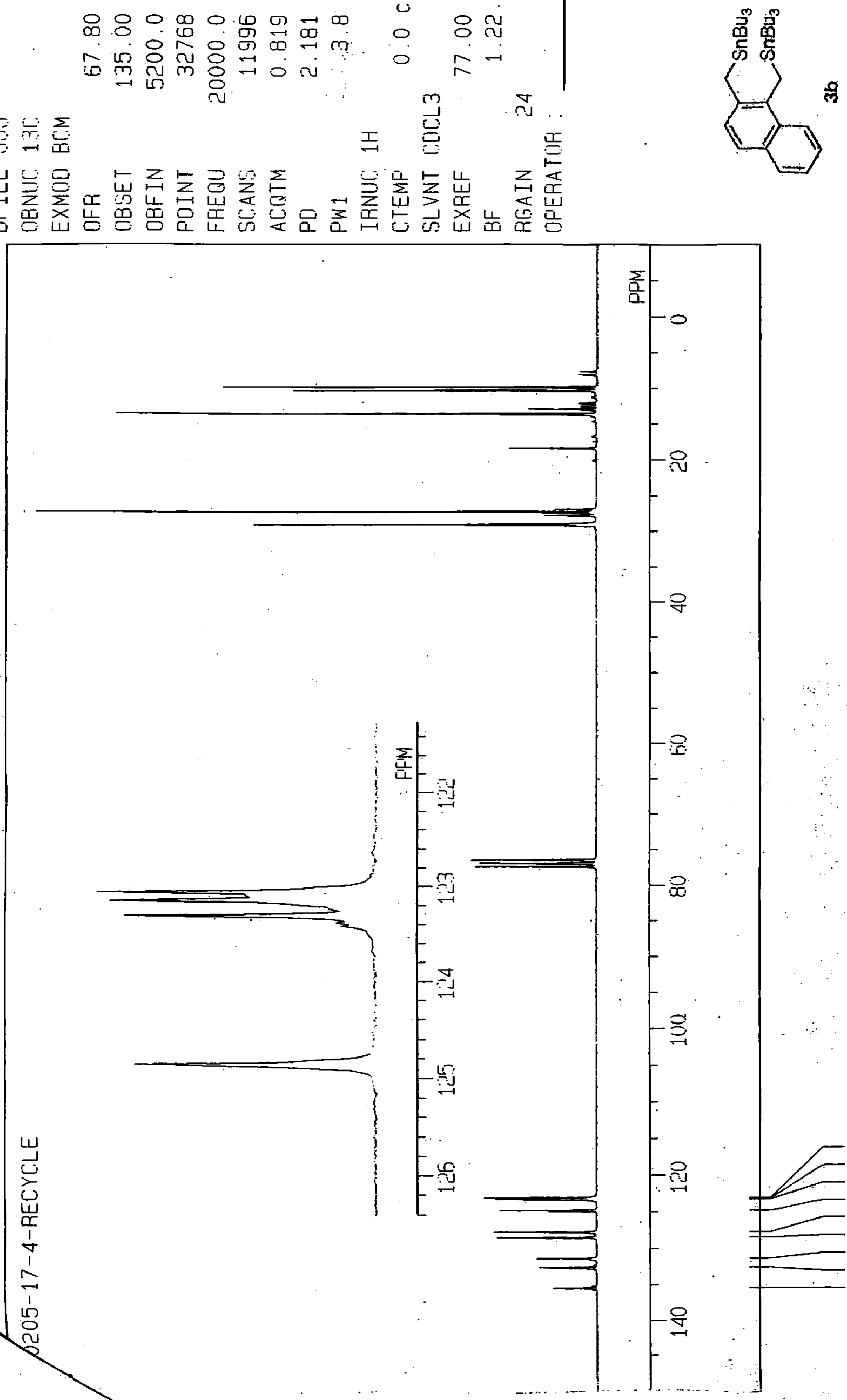


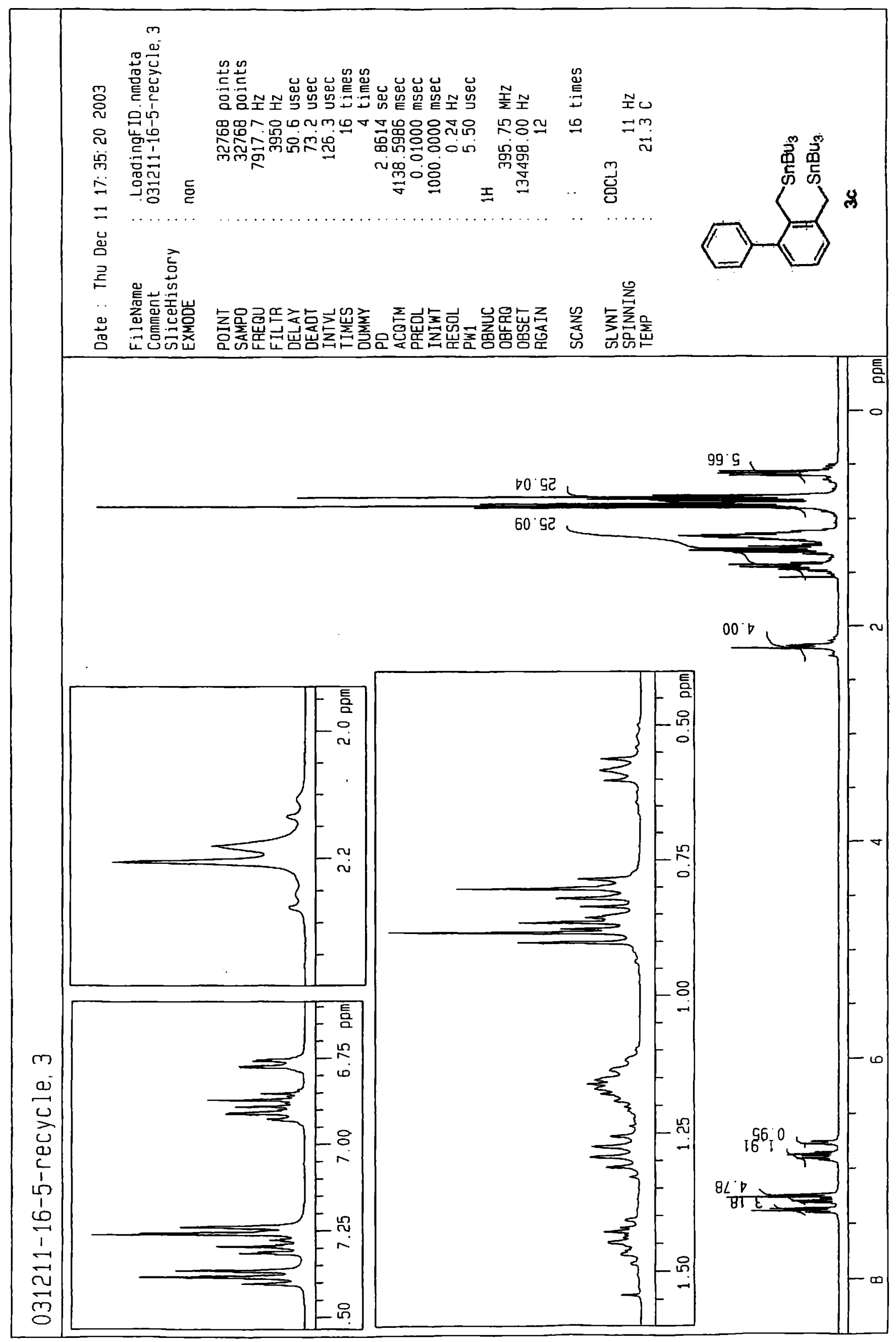




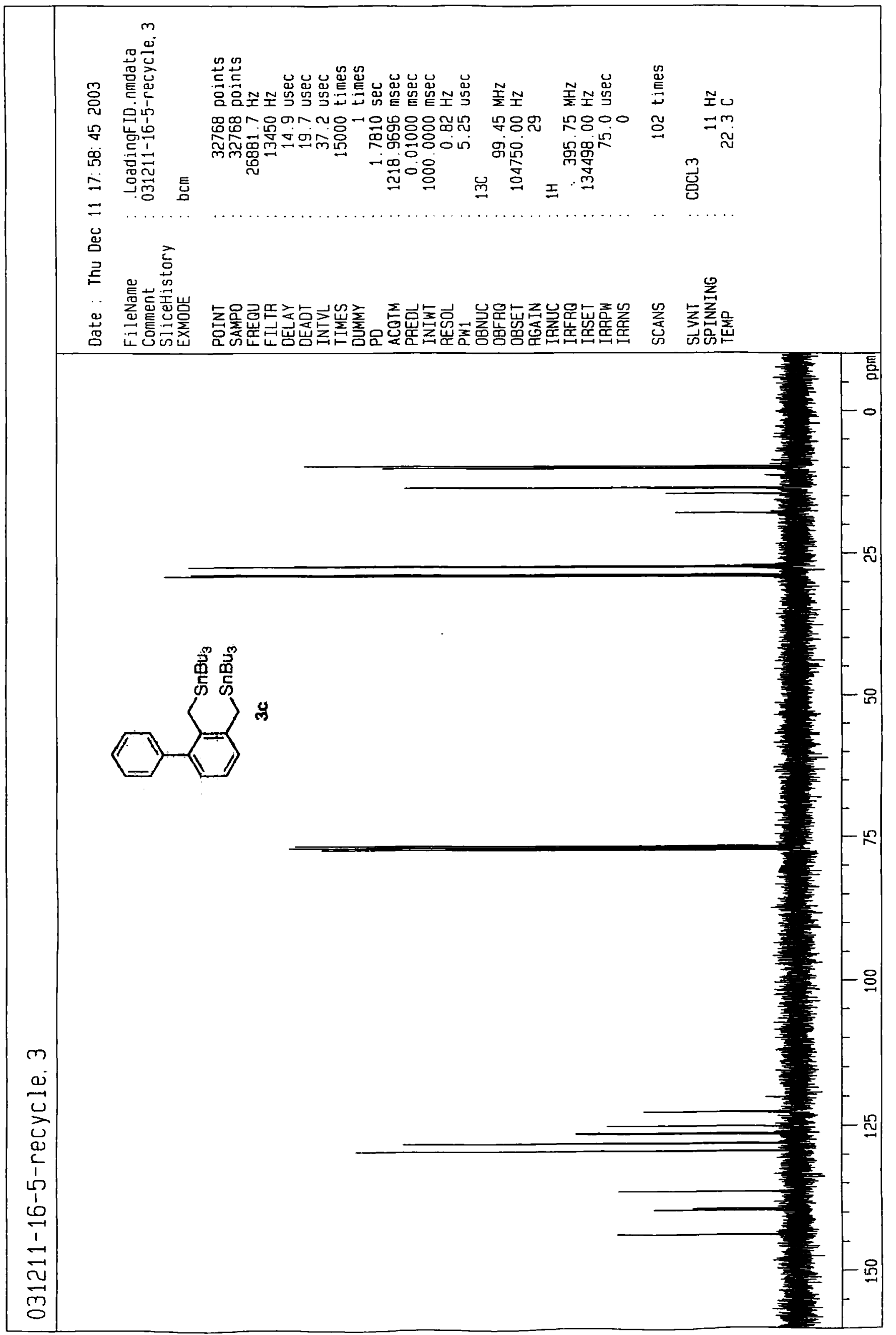




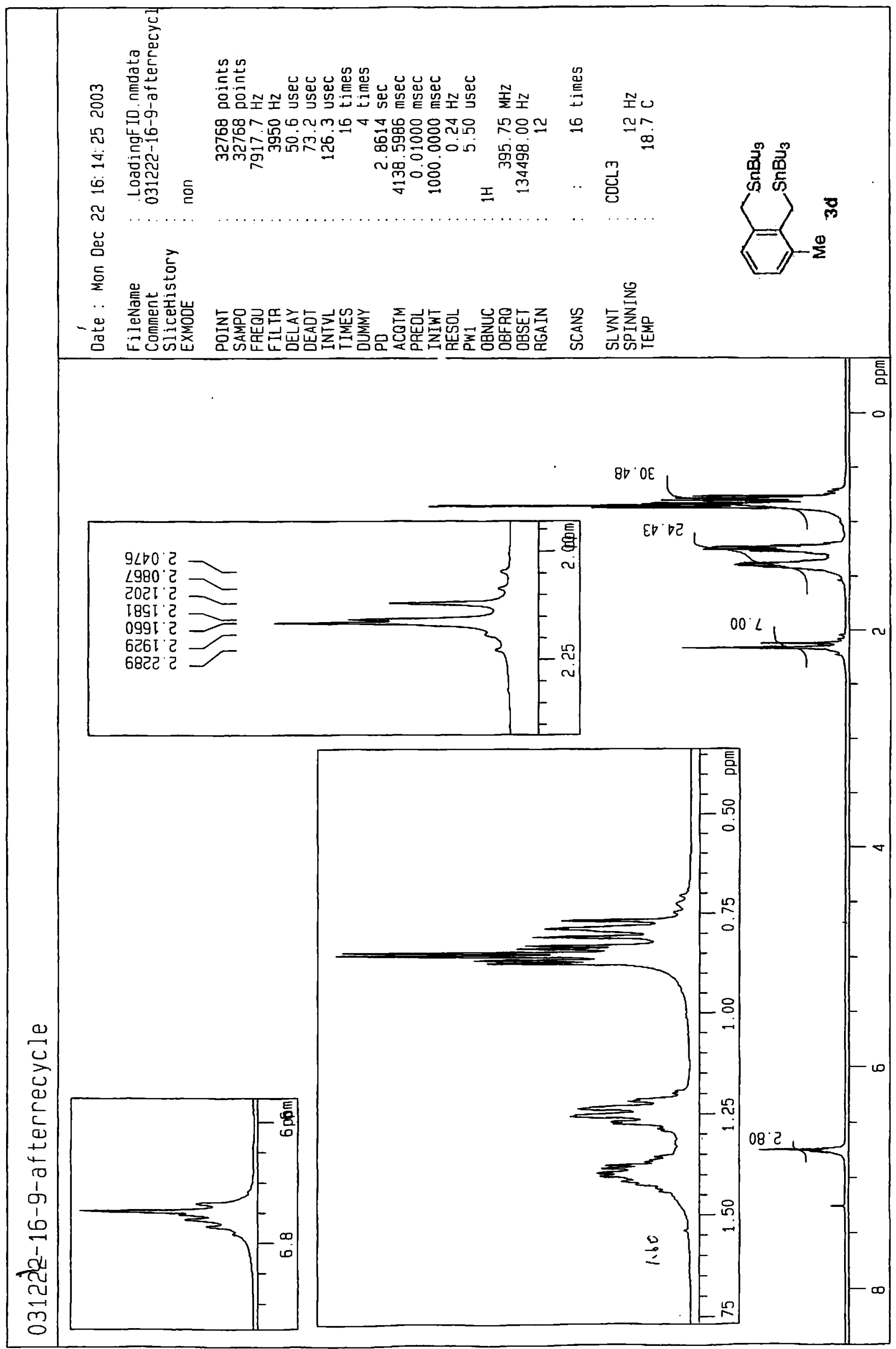




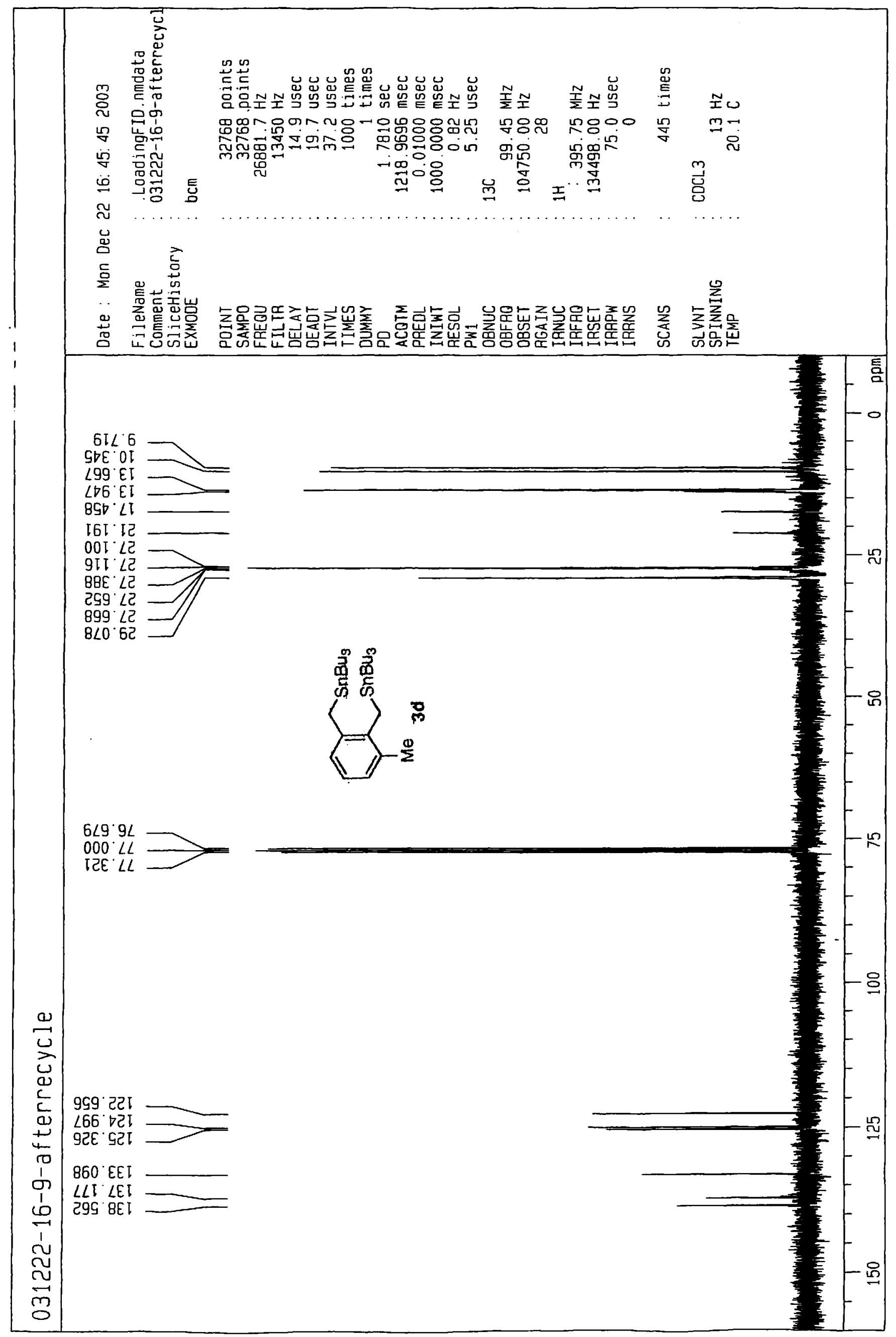



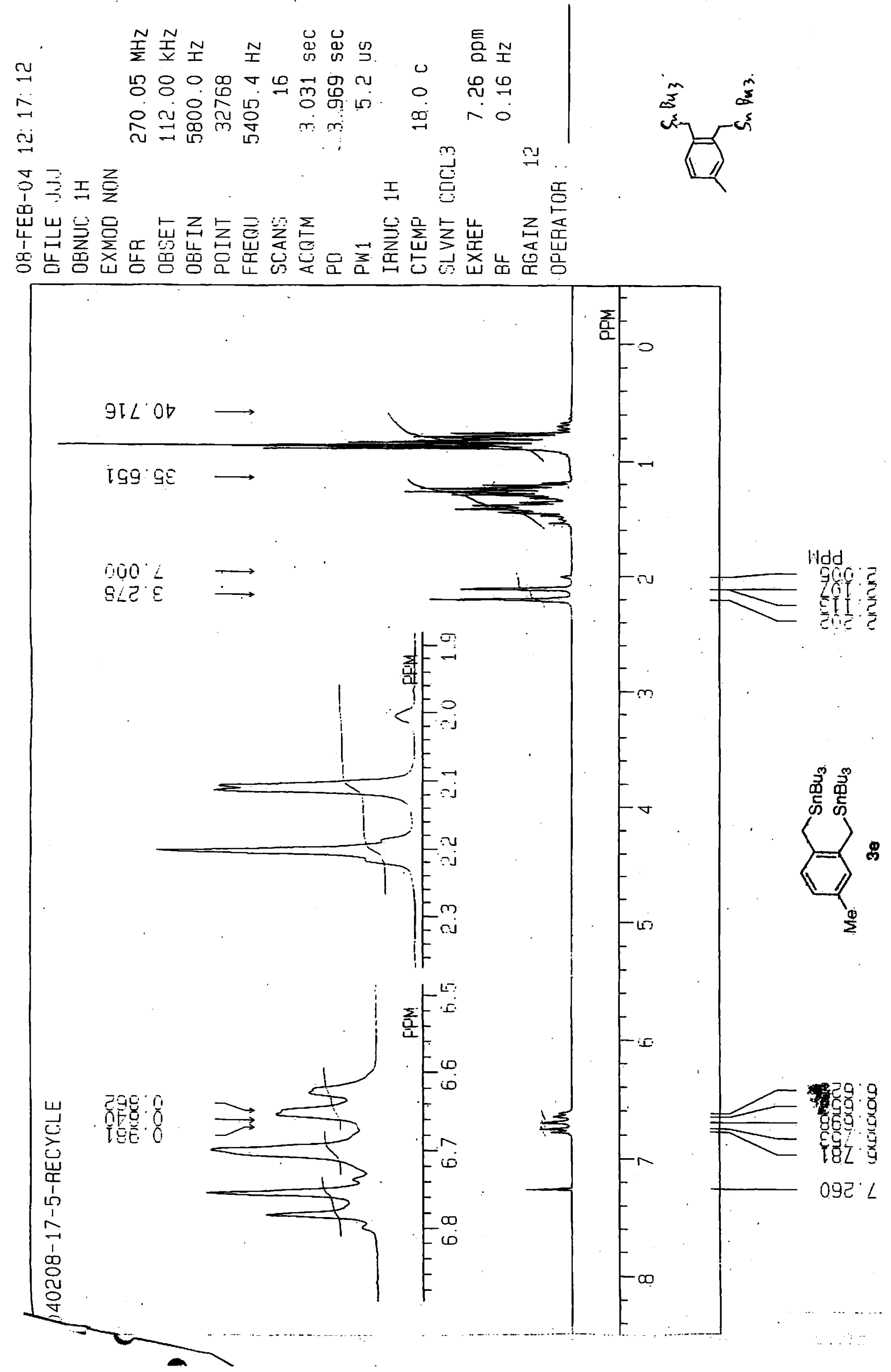


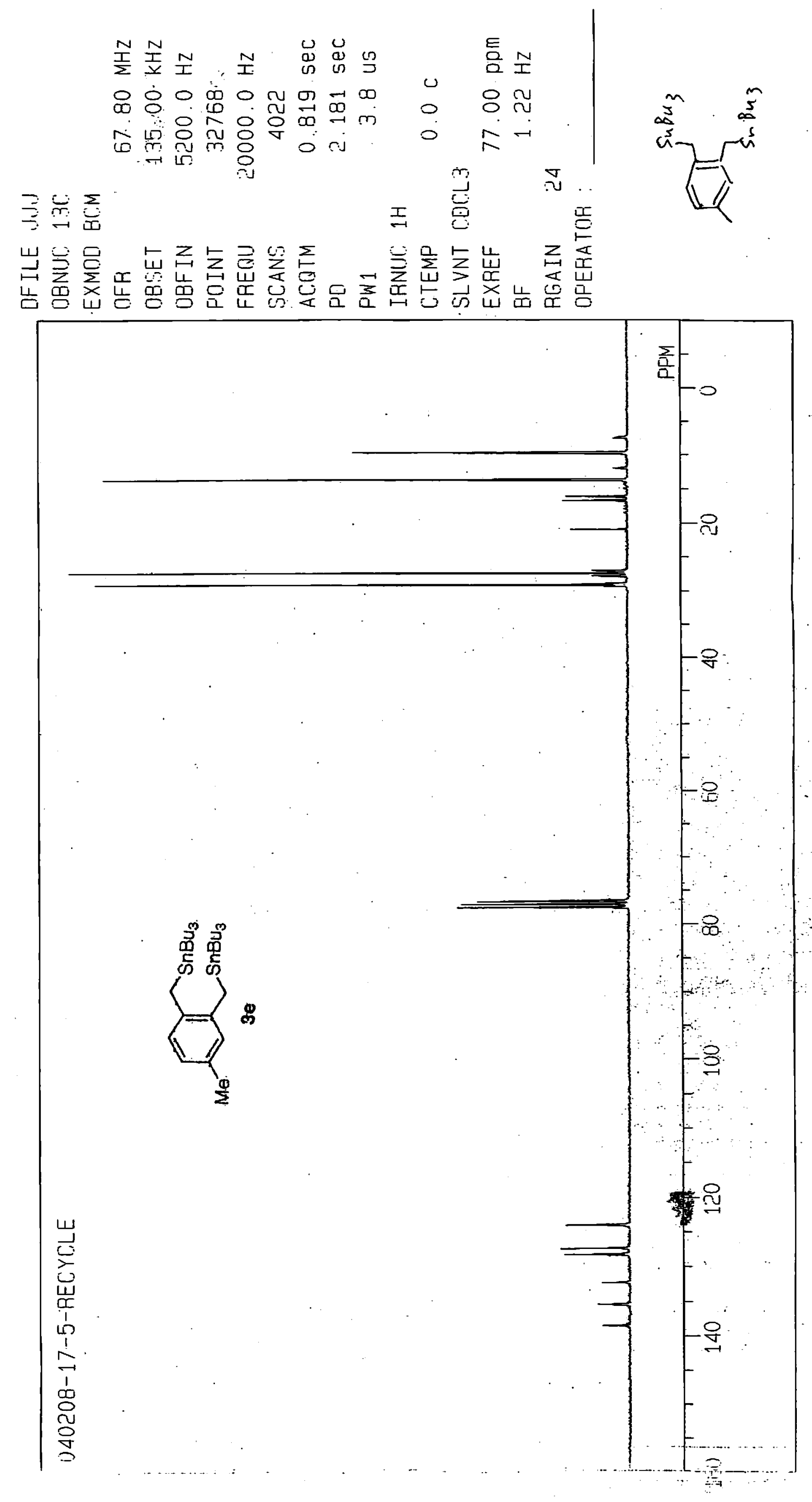




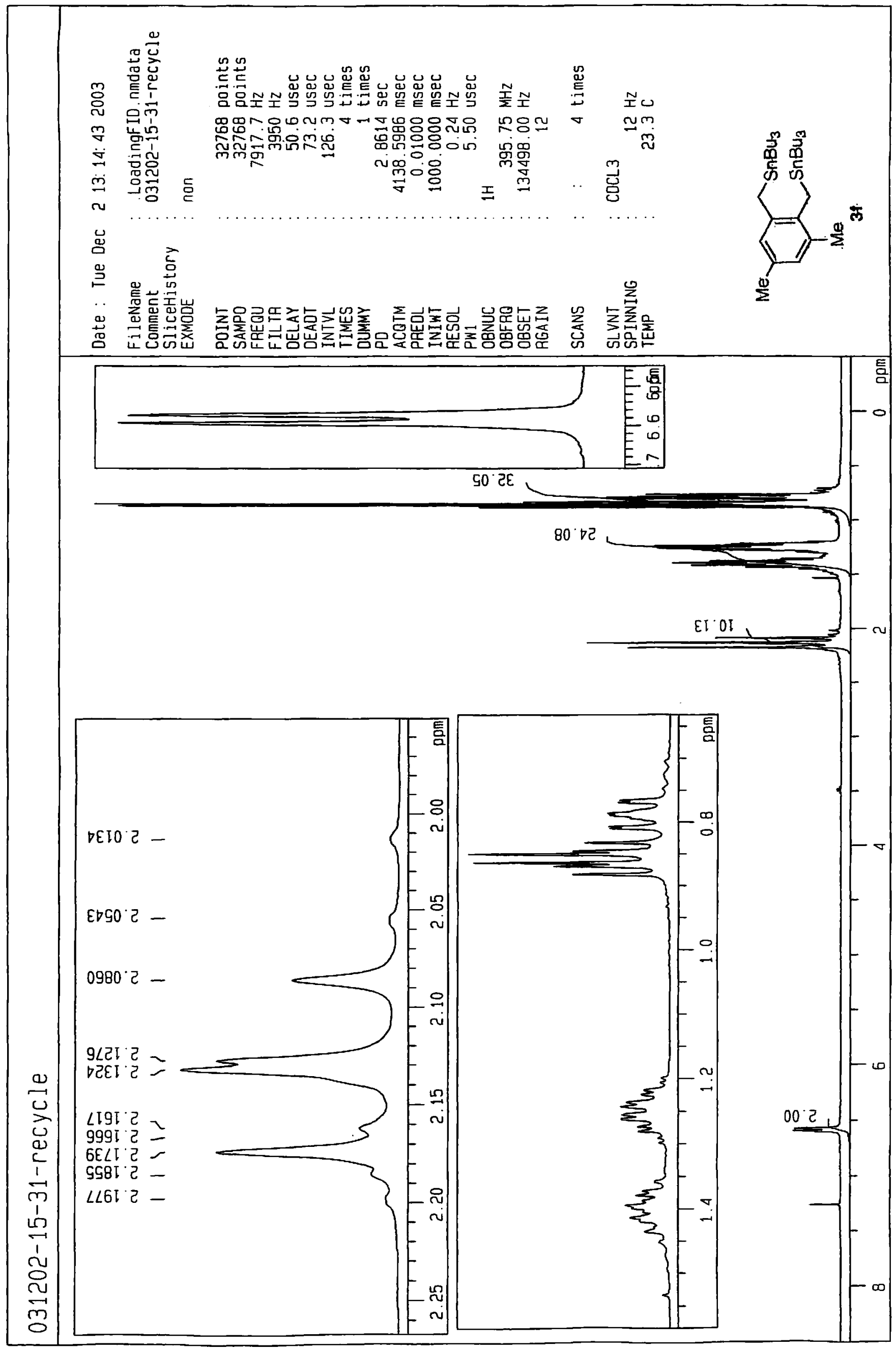




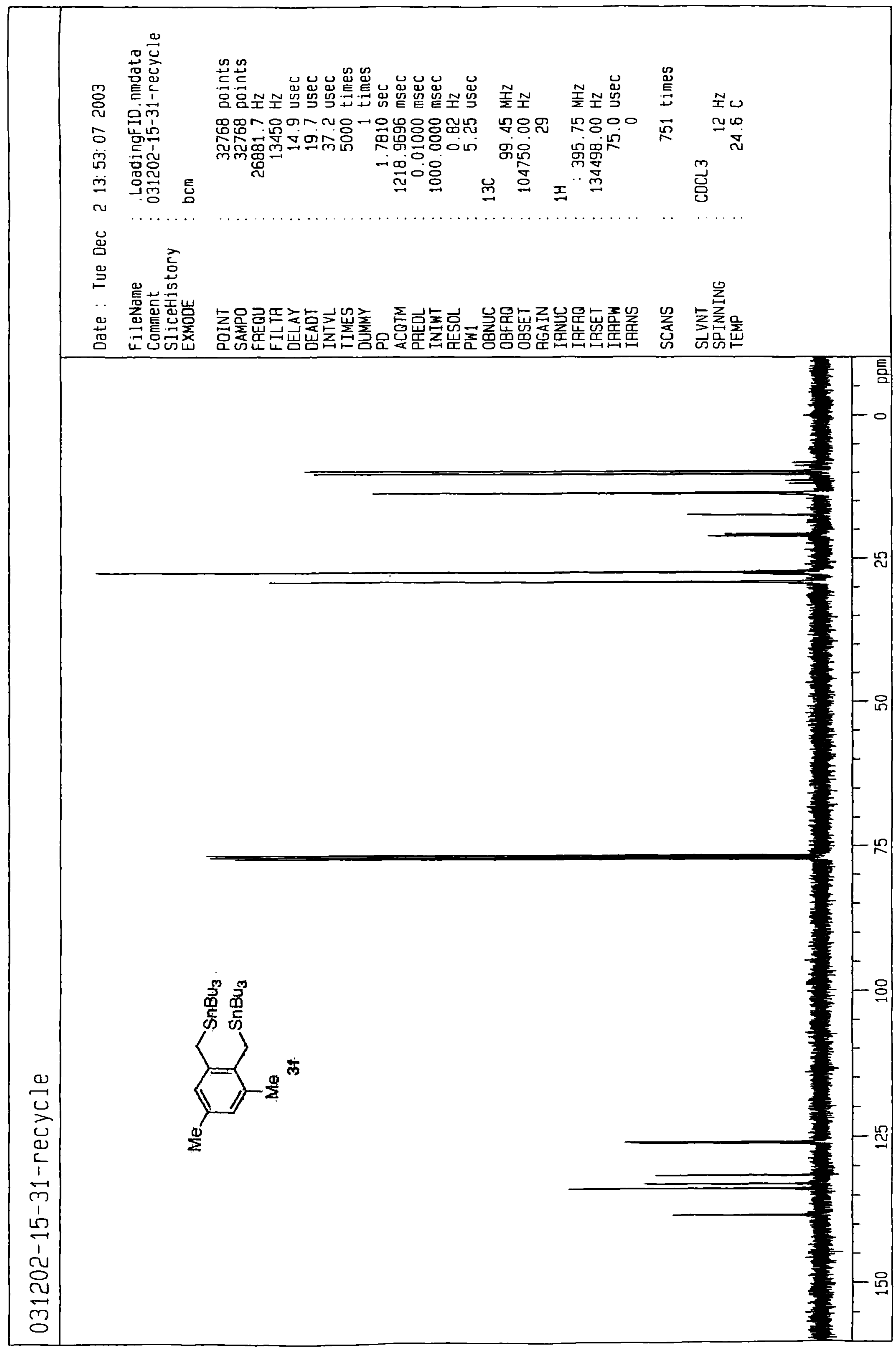




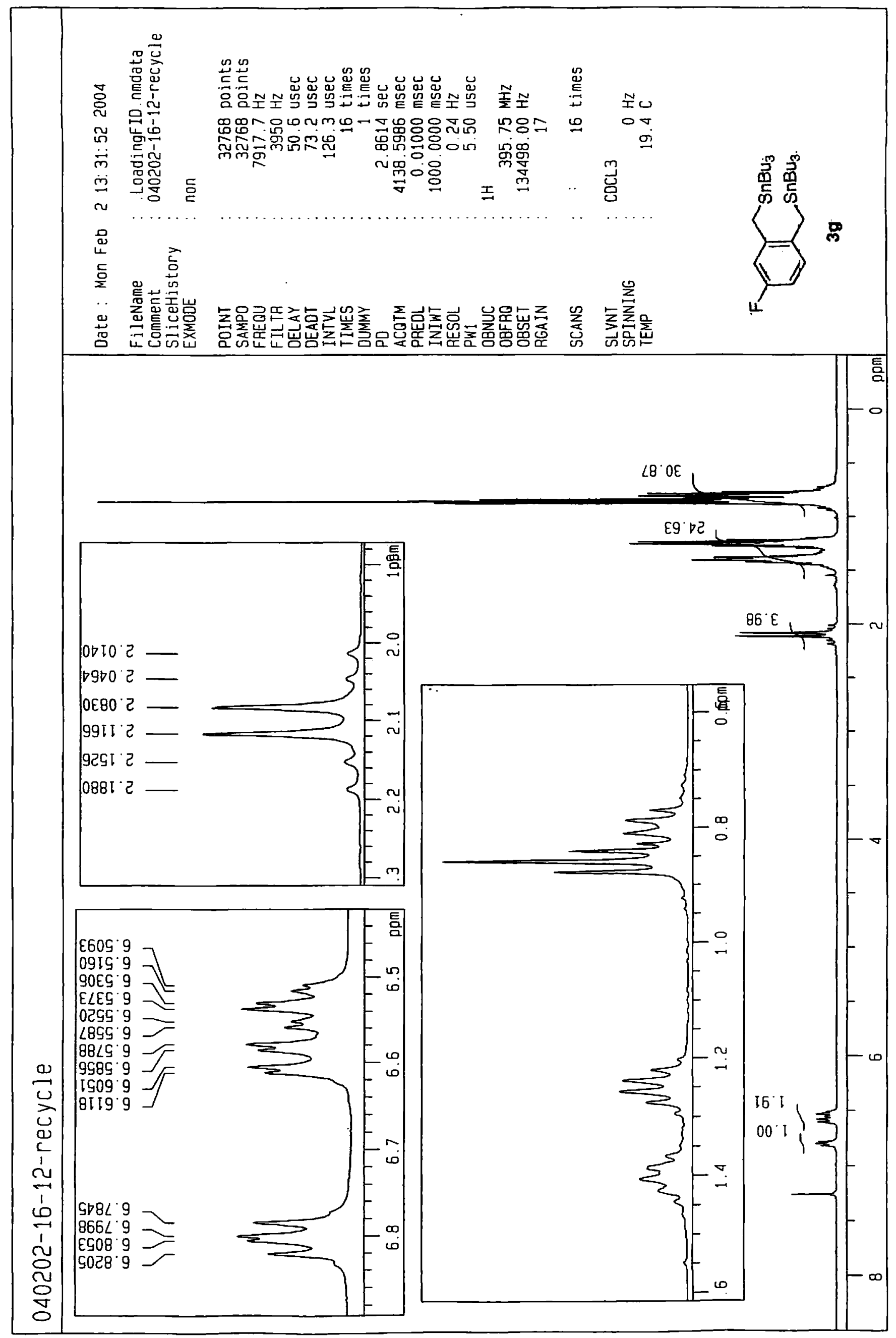




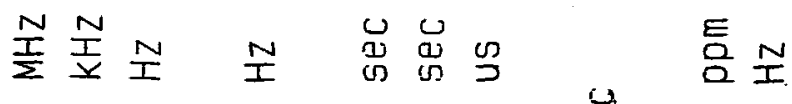

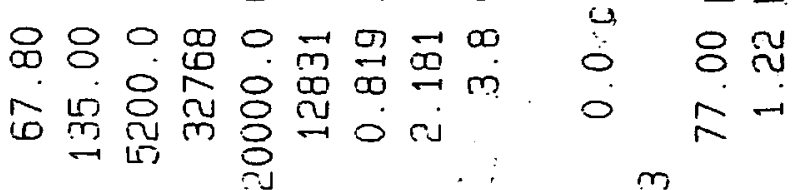

焉焉语

.

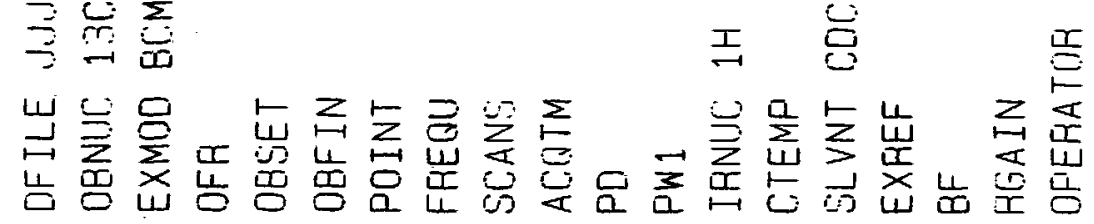

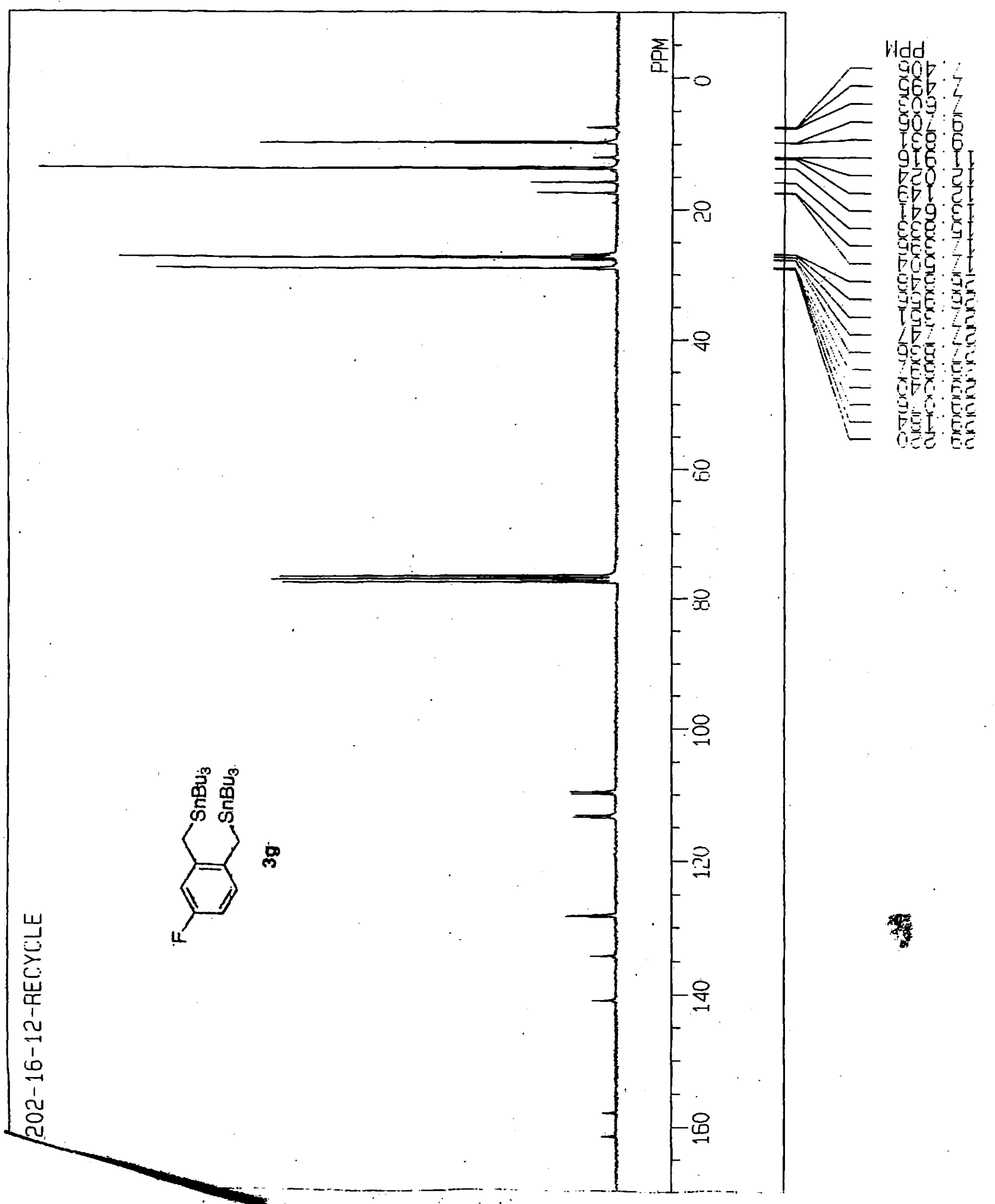




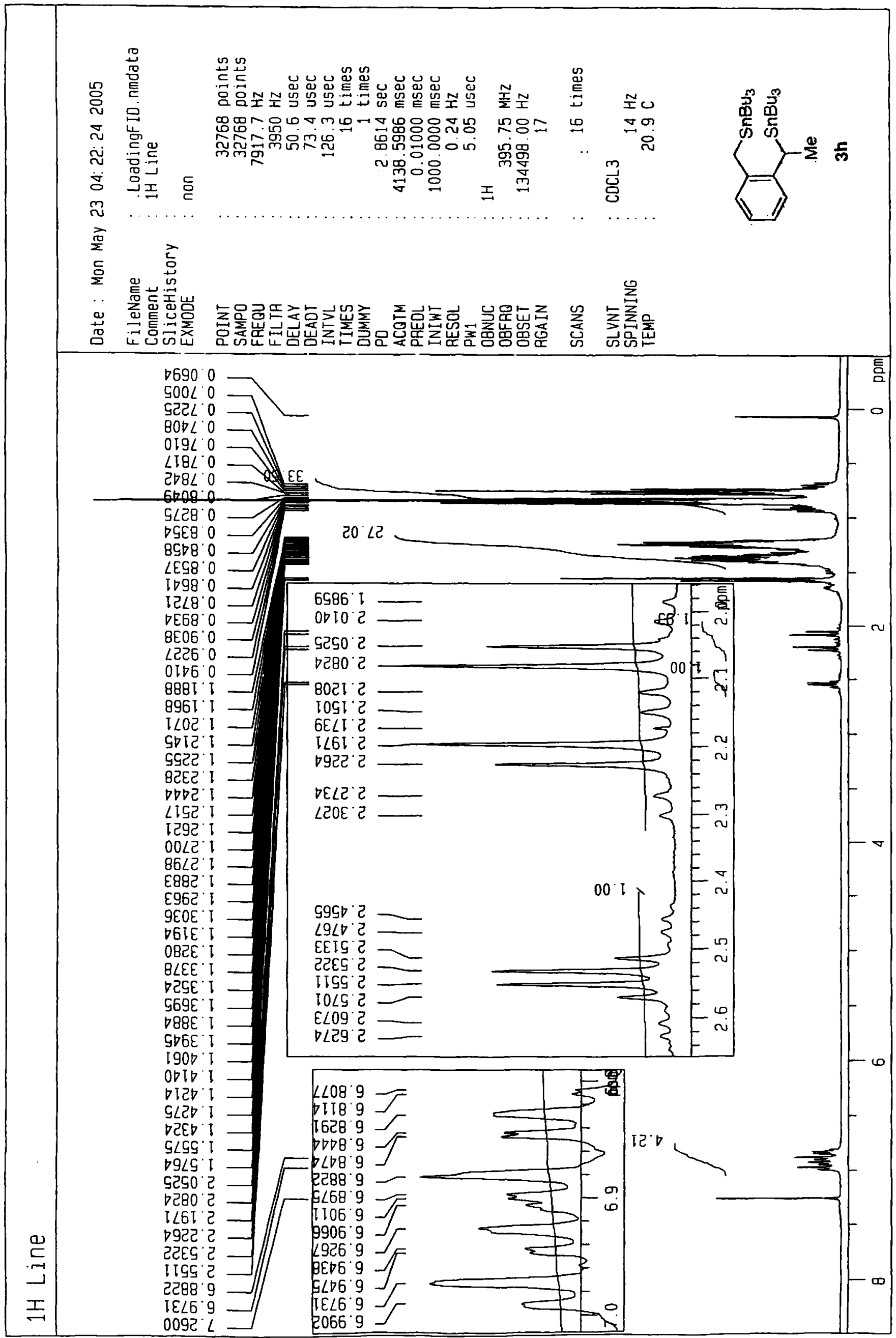




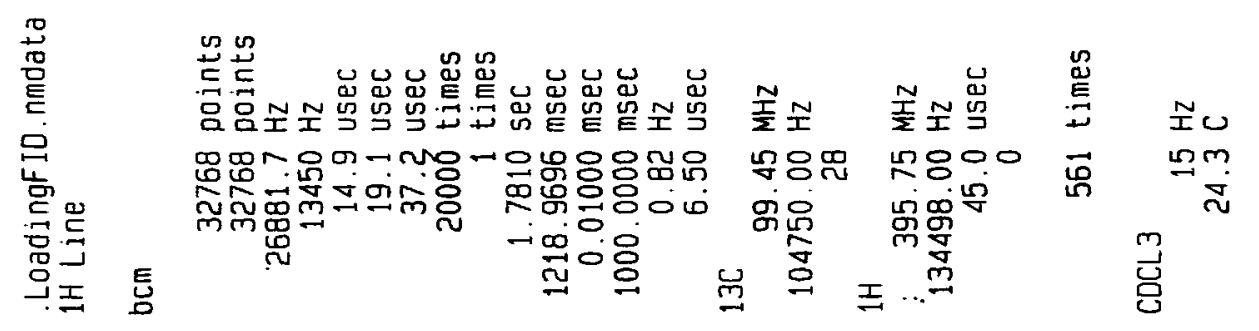

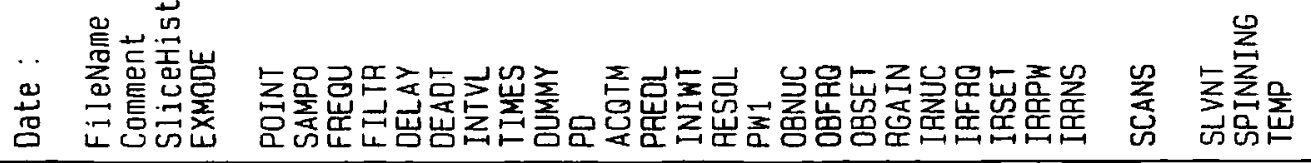

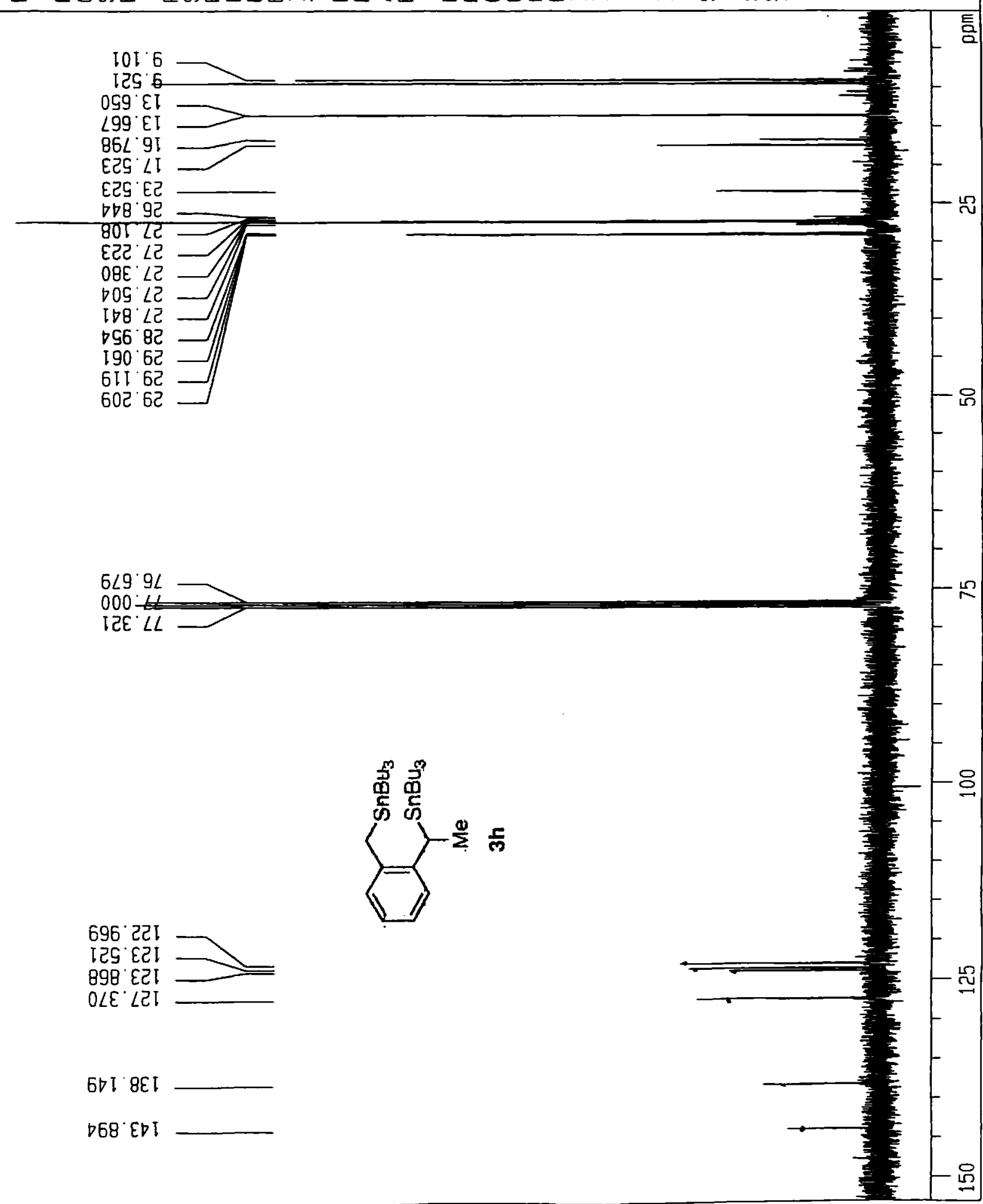




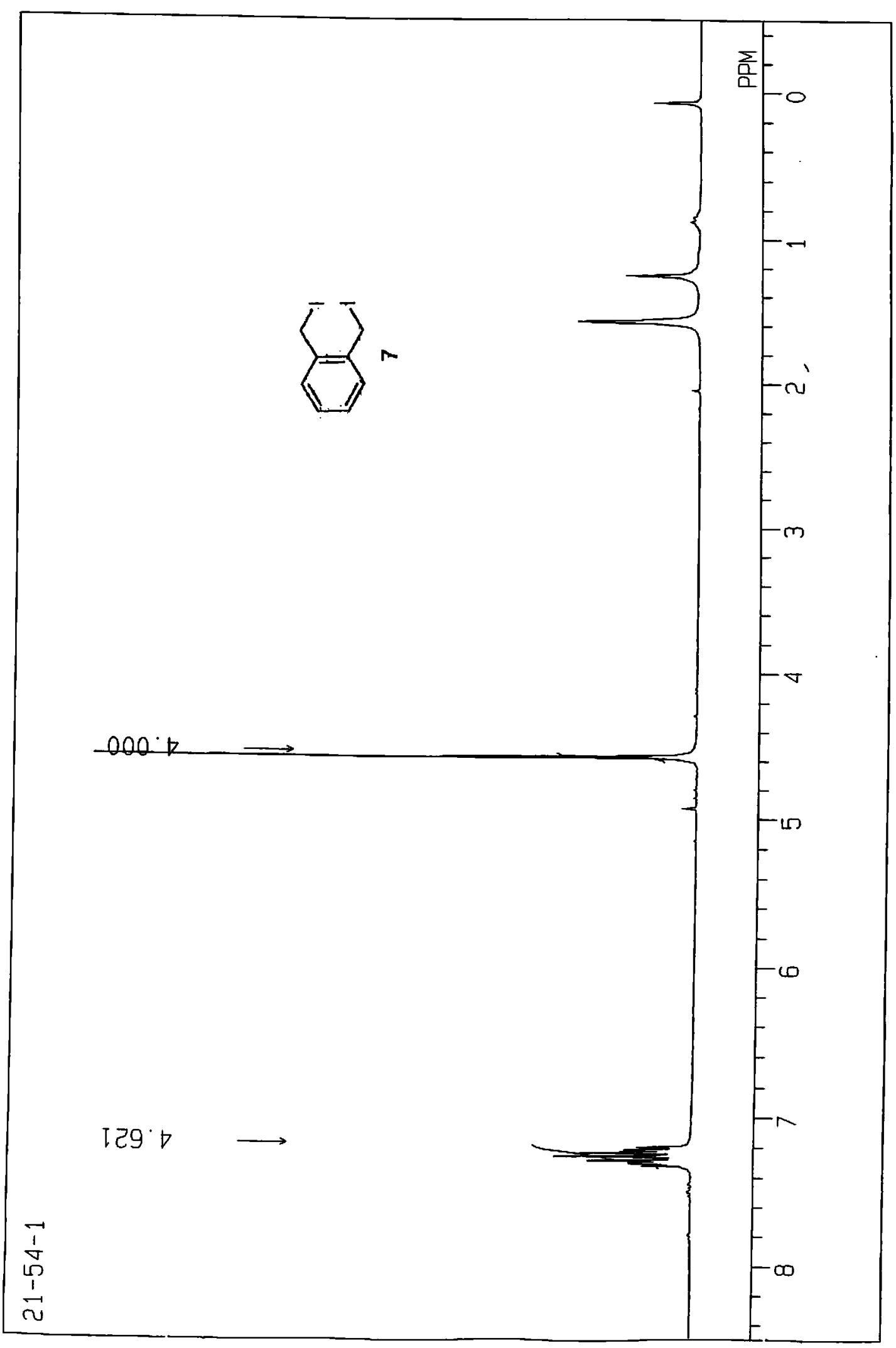




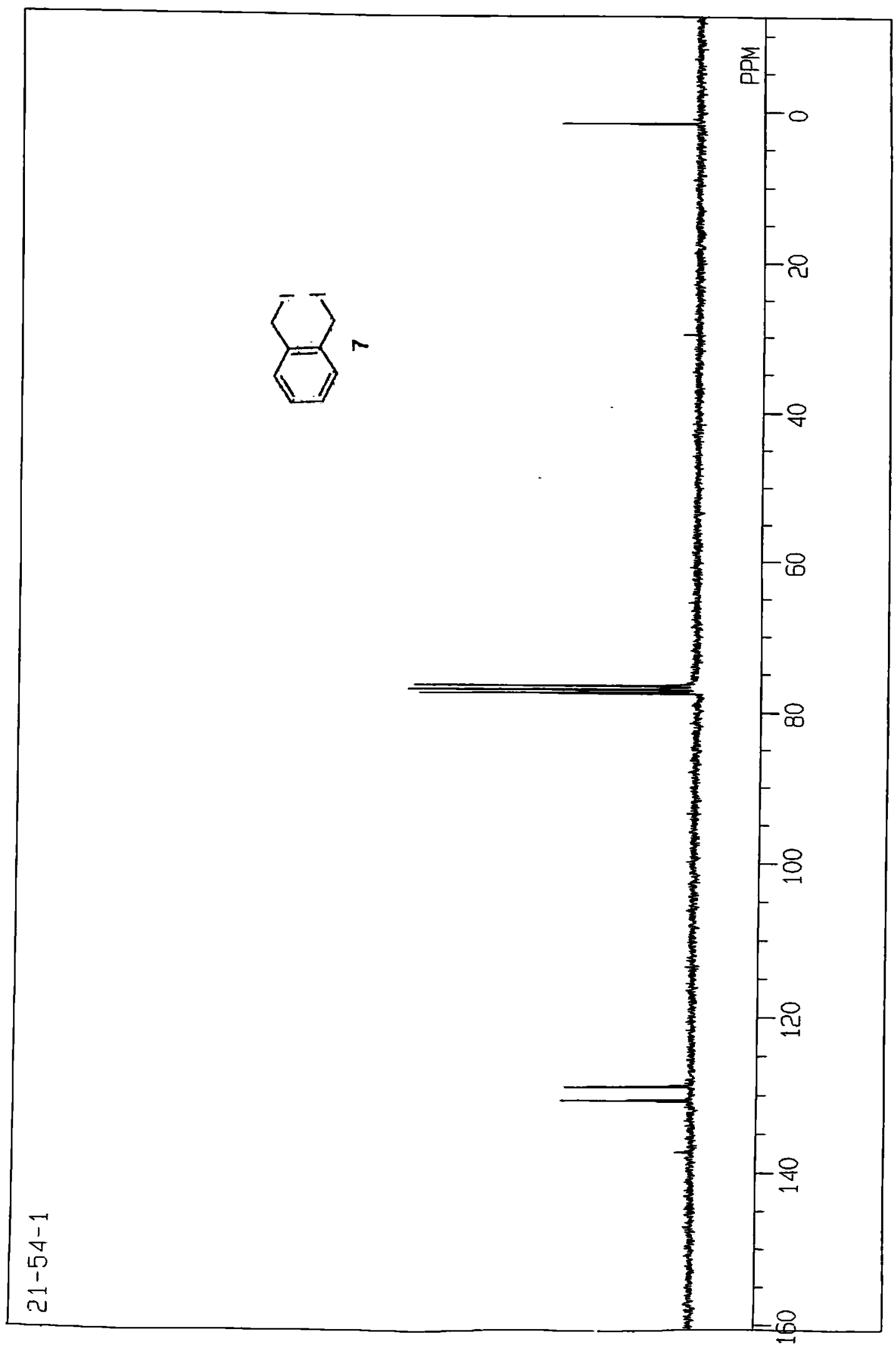



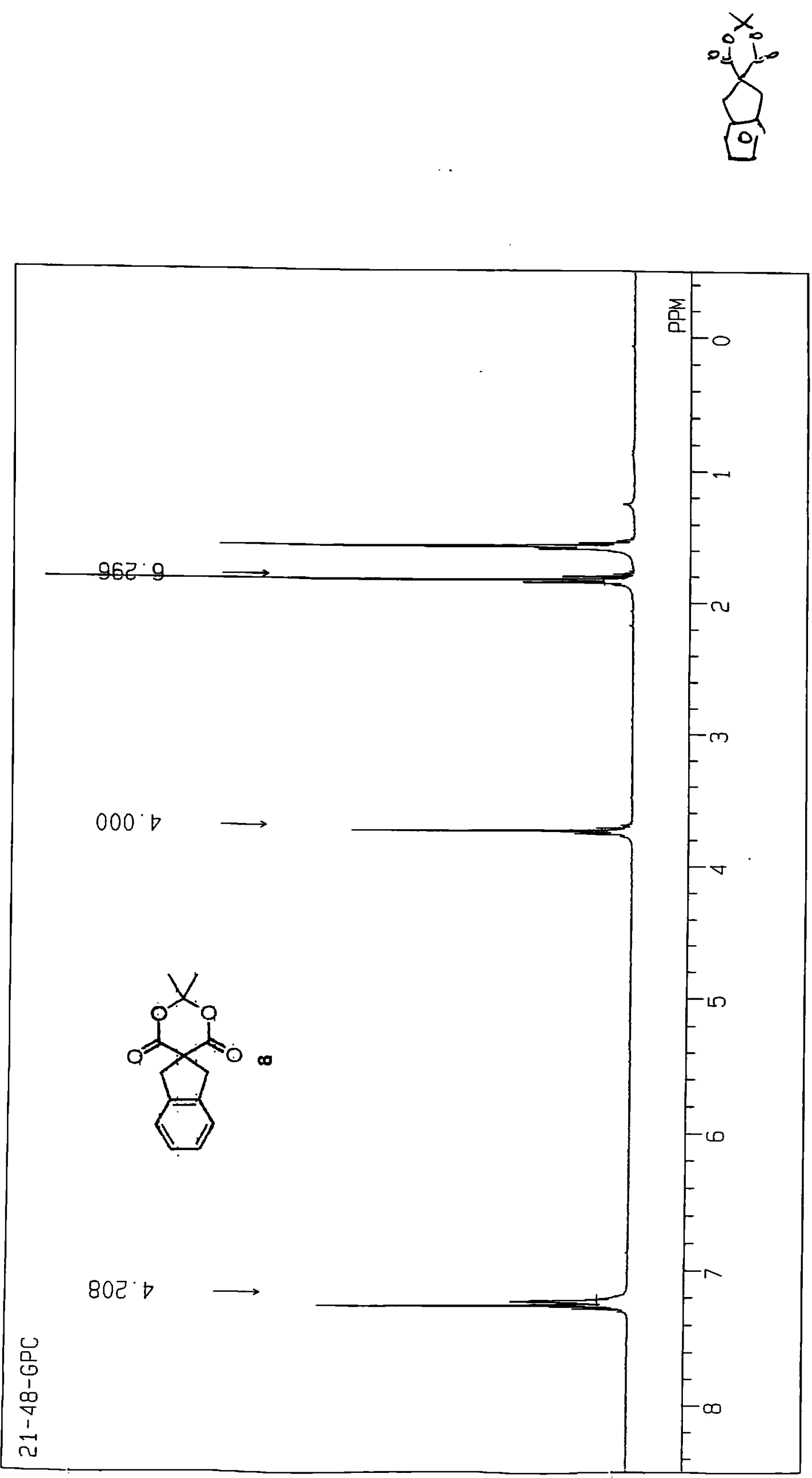

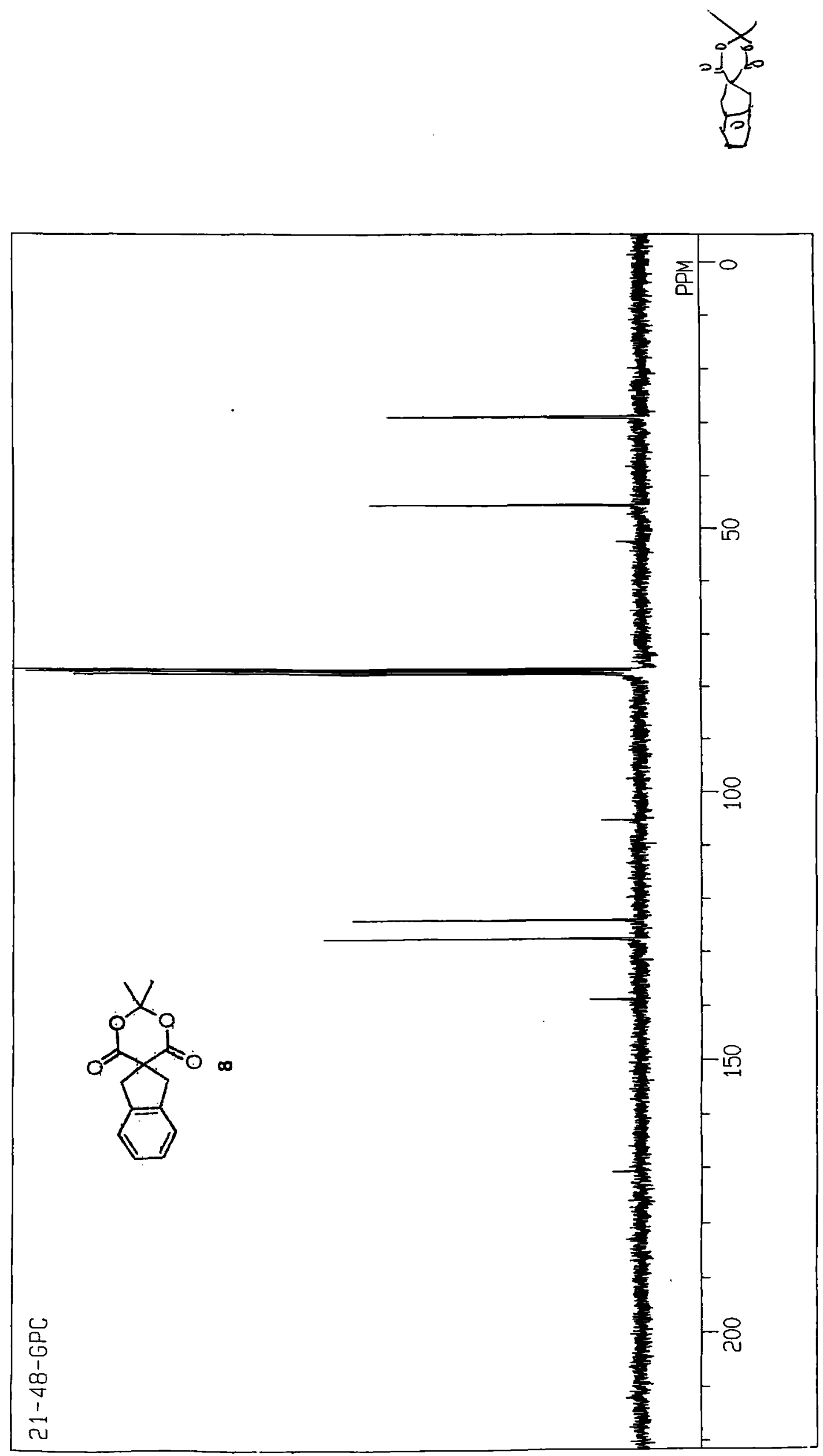


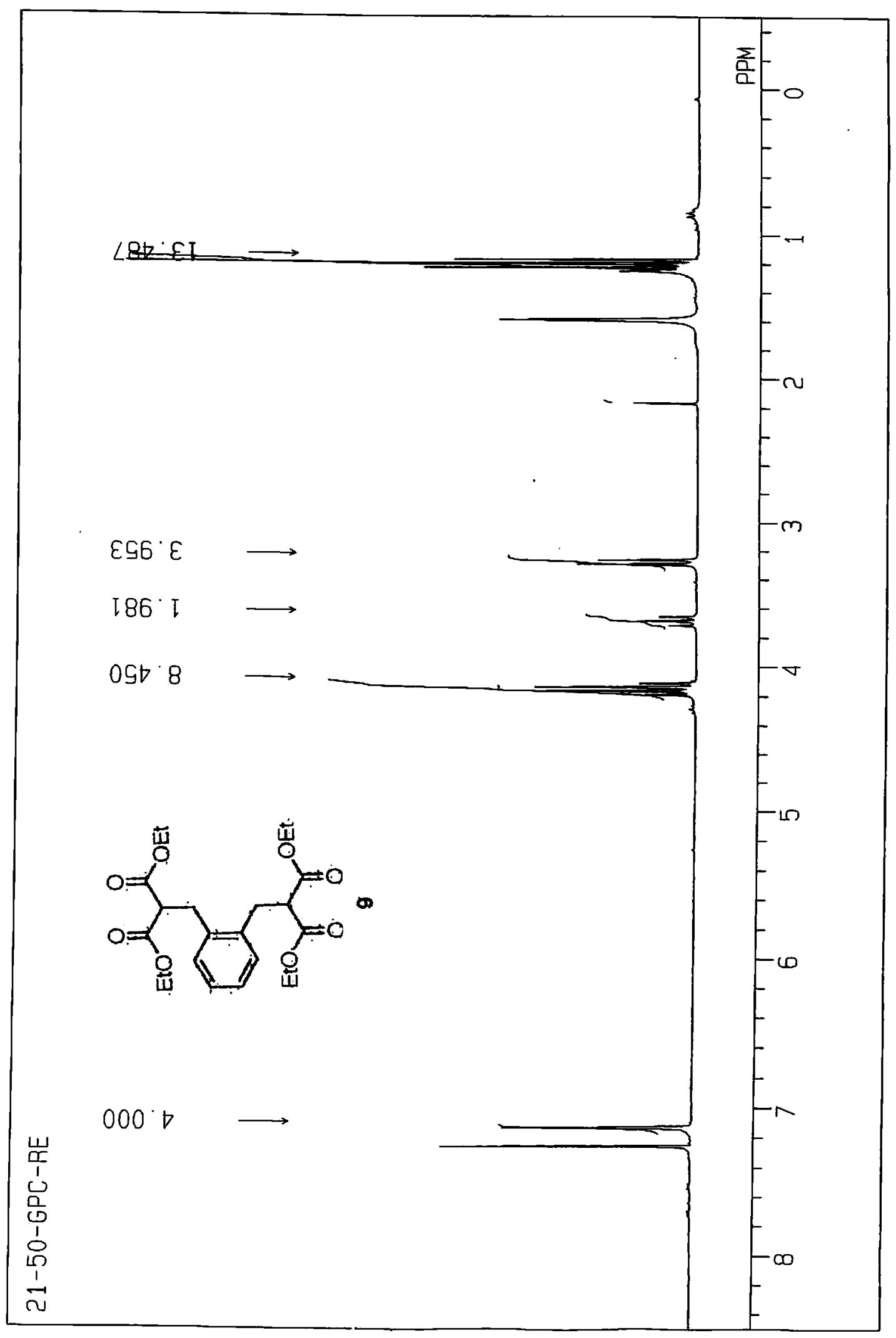




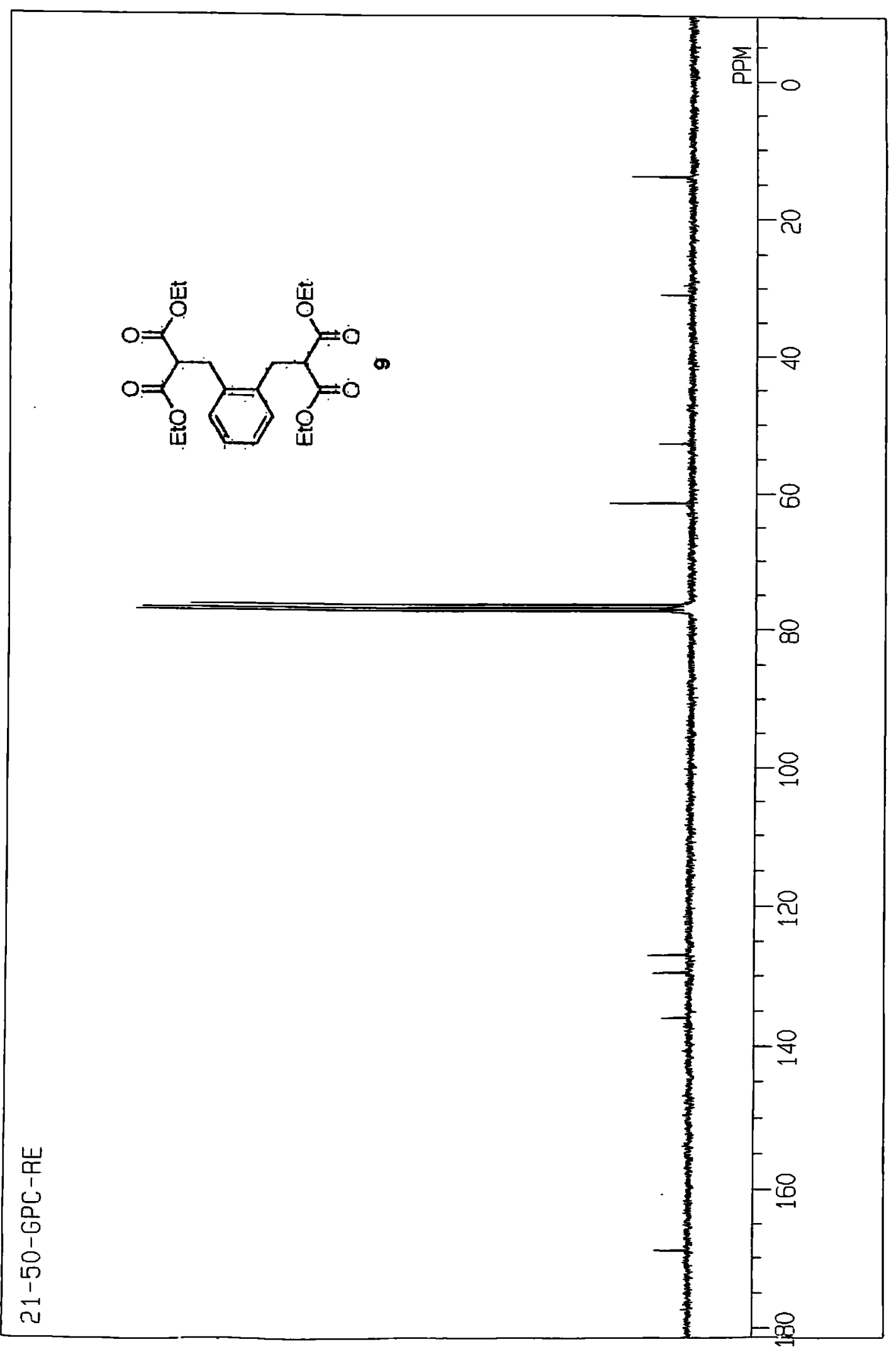

